\title{
UNBOUNDED FUNCTIONS IN THE UNIT DISC
}

\author{
L.R. SONS \\ Department of Mathematical Sciences \\ Northern Illinois University \\ DeKalb, Illinois 60115 U.S.A.
}

(Received June 24, 1982)

ABSTRACT. A survey is made of results related to the value distribution of functions which are meromorphic or analytic in the unit disc and have unbounded growth according to some specific growth indicator.

KEY WORDS AND PHRASES. Value distribution, unbounded functions, Nevanlinna theory. 1980 MATHEMATICS SUBJECT CLASSIFICATION CODE. 30.35

\section{INTRODUCTION}

We shall consider functions which are meromorphic or analytic in the unit disc $D=\{z|| z \mid<1\}$ and have unbounded growth according to some specific growth indicator. We give a survey of known results related to value distribution. Initially we shall consider functions whose growth in the disc makes them correspond to meromorphic functions of finite order in the plane. Then we shall proceed to functions with slower growth. We assume the notation of the elementary theory of Nevanlinna theory as it appears in Chapter One of Hayman's book [1].

2. SECTION TWO

First we consider functions which have their growth determined by the Nevanlinna characteristic function.

Let $f$ be a meromorphic function in $D$. We define the order $\alpha$ of $f$ by

$$
\lim _{r \rightarrow 1} \sup \frac{\log ^{+} T(r, f)}{-\log (1-r)}=\alpha
$$

where $T(r, f)$ is the Nevanlinna characteristic of $f$ at $r$. We note that $0 \leq \alpha \leq+\infty$. Further we define the lower order $\ell$ of $f$ by

$$
\lim _{r \rightarrow 1} \inf \frac{\log ^{+} T(r, f)}{-\log (1-r)}=\ell \text {, }
$$


and observe that $0 \leq \ell \leq \alpha \leq+\infty$.

In this case we have the following relationship between the order of a function and the order of its derivative function.

THEOREM 2.1. Let $f$ be a meromorphic function in $D$ with order $\alpha$ defined by (2.1). Let $\alpha^{\prime}$ be the order of $f^{\prime}$ defined by (2.1), and let $\ell$ and $\ell^{\prime}$ be the lower orders of $f$ and $f^{\prime}$, respectively, defined by (2.2). Thus $\alpha=\alpha^{\prime}$ (Tsuj1 [2] and $\left[3\right.$, p.228]) and $\ell^{\prime} \leq \ell$ (Sons [4]).

If $f$ is a meromorphic function in $D$ for which $\alpha$ defined by (2.1) is positive, we ask if $f$ must assume every value on the Riemann sphere and how frequently it may assume such values, An omitted value is called a Picard value,

THEOREM 2.2. Let $f$ be a meromorphic function in $D$ such that

$$
\lim _{r \rightarrow 1} \sup \frac{T(r, f)}{-\log (1-r)}=\infty \text {. }
$$

Let $g$ be a meromorphic function in $D$ with

$$
T(r, g)=0(1), \quad(r \rightarrow 1) .
$$

Then $f(z)-g(z)$ has infinitely many zero points in $D$ with two possible exceptions for 8. More precisely,

$$
\lim _{r \rightarrow 1} \sup \frac{N(r, 1 /(f-g))}{-\log (1-r)}=\infty
$$

with two possible exceptions for $g$ (Tsuji [5] and [3,p.297]).

Exceptions can indeed occur. For $k>1$ we define the functions $f_{k}$ in $D$ by

$$
f_{k}(z)=\exp \left((1-z)^{-k}\right) \text {. }
$$

We note that $f_{k}$ has order $\alpha=k-1$ and $f_{k}$ omits the values zero and infinity. A closer relationship between the growth of the function and the values assumed is given below.

TEEOREM 2.3. Let $f$ be a meromorphic function in $D$ with finite order $\alpha>0$ defined by (2.1). Let $\left\{a_{k}\right\}$ be a set of a-values for $f$ where $a$ is in $c u\{\infty\}$. Then

(i) $\quad \sum_{k}\left(1-\left|a_{k}\right|\right)^{\alpha+1+\varepsilon}<\infty$ for each $\varepsilon>0$;

(ii) $\sum_{k}\left(1-\left|a_{k}\right|\right)^{\alpha+1-\varepsilon}=\infty$ for each $\varepsilon>0$ with two possible exceptions for a; and 
(iii) when $\int_{\frac{1}{2}}^{1} T(r, f)(1-r)^{\alpha-1} d r=\infty$, we have $\Sigma\left(1-\left|a_{k}\right|\right)^{\alpha+1}=\infty$ with two possible exceptions for a (Tsuf1 [3, p,204 and 293] and [5]).

It can be further shown that if $f$ is a meromorphic function in $D$, then the convergence of

$$
\int_{\frac{1}{2}}^{1} T(r, f)(1-r)^{r-1} d r,
$$

for some $\gamma>0$, implies the finiteness of the three quantities

$$
\int_{\frac{1}{2}}^{1} N(r, a)(1-r)^{\gamma-1} d r, \int_{\frac{1}{2}}^{1} n(r, a)(1-r)^{\gamma} d r, \Sigma\left(1-\left|a_{k}\right|\right)^{\gamma+1},
$$

where $a$ is in $a \cup\{\infty\}$ and $\left\{a_{k}\right\}$ is the set of a-values for $f$. In fact, it can be shown that the three quantities in (2.4) converge or diverge simultaneously regardless of the known behavior of (2.3) (see Nevanlinna [6, Ch. X]).

If the $\left\{a_{k}\right\}$ are the non-zero zero points of $f$, it is convenient to define the convergence exponent $\mu \geq 0$ of the $\left\{a_{k}\right\}$ as follows.

If $\sum_{k}\left(1-\left|a_{k}\right|\right)<\infty$, then $\mu=0$.

If $\sum_{k}\left(1-\left|a_{k}\right|\right)=\infty$, then $\mu$ is that number such that $\underset{k}{\sum\left(1-\left|a_{k}\right|\right)^{\mu+1-\varepsilon}=\infty}$ and $\sum_{k}\left(1-\left|a_{k}\right|\right)^{\mu+1+\varepsilon}<\infty$

for any $\varepsilon>0$. From THEOREM 2.3 we see

$$
0 \leq \mu \leq \alpha
$$

if $\alpha$ is the order of $f$. We assume $\alpha$ is finite.

Let $p \geq 1$ be a positive integer such that

$$
\sum_{k}\left(1-\left|a_{k}\right|\right)^{p}=\infty \text { and } \sum_{k}\left(1-\left|a_{k}\right|\right)^{p+1}<\infty .
$$

If $\mu$ is not an integer, then $p=[\mu]+1$, and if $\mu$ is an integer then $p=\mu$ or $p=\mu+1$ according as $\sum_{k}\left(1-\left|a_{k}\right|\right)^{\mu+1}<\infty$ or $\sum_{k}\left(1-\left|a_{k}\right|\right)^{\mu+1}=\infty$. In any case,

$$
\mathrm{p}-1 \leq \mu \leq \alpha .
$$

Now we define the canonical product $P(z)$ formed with $\left\{a_{k}\right\}$ as below. If $\sum_{k}\left(1-\left|a_{k}\right|\right)<\infty$, then

$$
P(z)=\prod_{k=1}^{\infty}\left(1-\frac{1-\left|a_{k}\right|^{2}}{1-\bar{a}_{k} z}\right) .
$$

If $\sum_{k}\left(1-\left|a_{k}\right|\right)=\infty$, then 


$$
P(z)=\prod_{k=1}^{\infty}(1-A(k, z)) \exp \left(A(k, z)+\frac{1}{2}(A(k, z))^{2}+\ldots+\frac{1}{p}(A(k, z))^{p}\right)
$$

where

$$
A(k, z)=\frac{1-\left|a_{k}\right|^{2}}{1-\bar{a}_{k} z}
$$

We observe that $P$ is analytic in $D$ and $P\left(a_{k}\right)=0$ for $k=1,2,3, \ldots$. Further, when $P$ is defined by (2.5) we see

$$
|P(z)|<1, \quad(z \in D) \text {. }
$$

We then have two useful theorems.

THEOREM 2.4. Let $\mathrm{f}$ be a meromorphic function in $\mathrm{D}$ which has finite order $\alpha$. Let $\left\{a_{k}\right\}$ be the non-zero zero points of $f$, and let $P$ be the canonical product formed with $\left\{a_{k}\right\}$. Let $\alpha_{0}$ be the order of $P$. Then

$$
\alpha_{0}=\mu
$$

where $\mu$ is the convergence exponent of the $\left\{a_{k}\right\}$ (Tsuji $\left.[3, p .227\}\right)$.

THEOREM 2.5. Let $f$ be a meromorphic function with finite order $\alpha$ in $D$. Then $f(z)$ can be expressed in the form

$$
f(z)=\left(f_{1}(z)\right) /\left(f_{2}(z)\right), \quad(z \in D),
$$

where $f_{1}$ and $f_{2}$ are analytic functions in $D$ with order $\leq \alpha$ (Tsuj1 [3, p.227]). Using an extended notion of the characteristic function M. M. Dzhrbashyan [7] has introduced another factorization for functions meromorphic in D.

M. A. Girnyk has studied relationships between the growth of products of the form (2.6) and their value distribution. In [8] he has determined the asymptotic behavior of the logarithm of the modulus of products of the form (2.6) assuming the zeros are all positive and have an asymptotic behavior which is essentially

$$
n(r, 1 / f) \sim c(1-r)^{-\rho}
$$

for $0<\mathrm{C}<\infty, \rho>0$. In [9] Girnyk discusses the asymptotic behavior of the Nevanlinna characteristic and the logarithm of the modulus of products of the form (2.6) in the case when the zeros possess an angular density. He gives an example to show that even in the case of nonintegral order, the angular density of the zeros does not uniquely determine the asymptotics (so his theorems require some additional restrictions). 
The parts (i) and (1i) of THEOREM 2.3 and the relationship among the quantities in (2.4) lead us to define Borel exceptional value as follows.

Let $f$ be a meromorphic function in $D$ and define $\mu_{a}$ for each $a$ in $a$ by

$$
\lim _{k \rightarrow 1} \sup \frac{\log N(r, a)}{-\log (1-r)}=\mu_{a}
$$

By the First Fundamental Theorem of Nevanlinna theory we have $N(r, a) \leq T(r, f)+0(1)$, $(r \rightarrow 1)$, and so $0 \leq \mu_{a} \leq \alpha . \mu_{\infty}$ is defined similarly. If $\alpha<+\infty$, we say a is a Borel exceptional value for $\mathrm{f}$ if $\mu_{a}<\alpha$. If $\alpha=\infty$, we say a is a Borel exceptional value for $f$ if $\mu_{a}<\infty$.

THEOREM 2.6. Let $f$ be a meromorphic function in $D$ such that $f$ has order $\alpha \leq \infty$. Then for every a in $\mathbb{\alpha} \cup\{\infty\}$ with at most two exceptions, $\mu_{a}=\alpha$ (c.f. Sons [10]).

Define $\bar{N}(r, a)$ in a similar manner to $N(r, a)$ where we consider only the distinct a-values of $f$. Let

$$
\lim _{r \rightarrow 1} \sup \frac{\log \bar{N}(r, a)}{-\log (1-r)}=\bar{\mu}_{a} \text {. }
$$

THEOREM 2.7. Let $f$ be a meromorphic function in $D$ such that $f$ has order $\alpha \leq \infty$. Then for every a in $\boldsymbol{\alpha} \cup\{\infty\}$ with at most two exceptions, $\bar{\mu}_{a}=\alpha$ (c.f. Sons [10]).

In the same sphere of ideas as THEOREMS 2.6 and 2.7 results may be obtained (c.f. Sons [10]) concerning the counting function for simple zeros, and also one may be obtained involving the counting function for distinct simple and double zeros.

The function $f$ defined in $D$ by $f(z)=\exp \left((1-z)^{-3 / 2}\right)$ has zero and $\infty$ as Borel exceptional values and order $\alpha=\frac{1}{2}$. Thus a function defined in $D$ with a Borel exceptional value need not have integer order.

Another type of measure of the values a function assumes is the Nevanlinna deficiency -- which is defined using a ratio of the counting iunction $N$ to the Nevanlinna characteristic T. A transitional result in this direction is below.

THEOREM 2.8. Let $\left\{a_{k}\right\}$ be a sequence of non-zero complex numbers with moduli less than one such that

$$
\sum_{k}\left(1-\left|a_{k}\right|\right)=\infty,
$$

but the convergence exponent $\mu$ of the $\left\{a_{k}\right\}$ satisfies $\mu<+\infty$. Let $P$ be a canoni- 
cal product formed with $\left\{a_{k}\right\}$, and let $\alpha$ be the order of $P$. Then

(1) $\alpha=\mu_{0}=\mu$;

(ii) when $p \neq \alpha$, there is a positive constant $c$ such that

$$
\lim _{r \rightarrow 1} \sup \frac{N(r, 1 / P)}{T(r, P)} \geq c,
$$

where $c$ depends only on $\alpha$ and $p$ (Sons [11]),

Part (i) is also considered in M.A. Girnyk [8].

If $f$ is a meromorphic function in $D$ and $a$ is in $\mathbb{C} \cup\{\infty\}$, then the First Fundamental Theorem of Nevanlinna theory says

$$
N(r, a)+m(r, a)=T(r, f)+0(1),(r \rightarrow 1) \text {. }
$$

This theorem relating the behavior of $m(r, a)$ to $T(r, f)$ also can give information about value distribution.

THEOREM 2.9. Let $f$ be a meromorphic function in $D$ such that

$$
\lim _{r \rightarrow 1} T(r, f)=+\infty
$$

Then for almost all a,

$$
\underset{r \rightarrow 1}{\lim \sup _{r}} \frac{m(r, a)}{\log T(r, f)} \leq 1 / 2 .
$$

The above theorem was proved by J.E. Littlewood [12]. This theorem and some related results of L. Ahlfors [13] are stated in W,K. Hayman [14] where examples are given to show their best possible character.

If $f$ is a meromorphic function in $D$ and $a$ is in $\mathbb{C}\{\{\}$ we define:

$$
\begin{aligned}
& \delta(a)=\delta(a, f)=\lim _{r \rightarrow 1} \inf \frac{m(r, a)}{T(r, f)}=1-\lim _{r \rightarrow 1} \sup \frac{N(r, a)}{T(r, f)} \\
& \Theta(a)=\Theta(a, f)=1-\lim _{r \rightarrow 1} \sup \frac{\bar{N}(r, a)}{T(r, f)}, \\
& \Theta(a)=\theta(a, f)=\lim _{r \rightarrow 1} \inf \frac{N(r, a)-\bar{N}(r, a)}{T(r, f)}
\end{aligned}
$$

$\delta(a)$ is called the Nevanlinna deficiency of a and $\theta(a)$ is called the index of multiplicity of a. It is clear from the definition of $\delta(a)$ that $0 \leq \delta(a) \leq 1$, and if a is a Picard value, then $\delta(a)=1$. Some functions may have a number of deficient values, but for the class of functions under consideration there are some restrictions on the number and their size. 
THEOREN 2.10, Let $f$ be a meromorphic function in $D$ such that

$$
\lim \sup \frac{T(r, f)}{-\log (1-r)}=+\infty
$$

Then the set of values $a$ in $\boldsymbol{d} \cup\{\infty\}$ for which $\Theta(a)>0$ is countable, and summing over all such values a, one gets

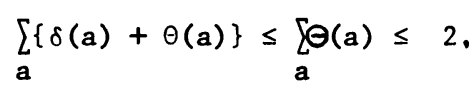

If $f$ is a function defined in $D$ by $f(z)=\exp \left\{(1-z)^{-2}\right\}$, then $\delta(0, f)=$ $\delta(\infty, f)=1$, so THEOREM 2.10 has two as a best possible bound.

It is natural to ask whether functions exist which have an "arbitrary" assignment of deficiencies at an arbitrary sequence of complex numbers subject only to the conditions of THEOREM 2.10. We state two results in this direction.

THEOREY 2.11. Let $\alpha$ be a non-negative real number, and let $\left\{\delta_{k}\right\}$ be a sequence of positive numbers less than or equal to one satisfying

$$
\sum_{k=1}^{\infty} \delta_{k} \leq 2 \text {. }
$$

Let $\left\{a_{k}\right\}$ be a sequence of distinct complex numbers. Then there is a meromorphic function $f$ in $D$ with order $\alpha$ such that $\delta\left(a_{k}, f\right) \geq\left(\delta_{k}\right) / 4, k=1,2,3, \ldots$. (Krutin [15]).

THEOREM 2.12. Let $\left\{\delta_{\mathrm{k}}\right\}$ be a sequence of positive numbers such that

$$
\sum_{k=1}^{\infty} \delta_{k} \leq 1 \text {. }
$$

Let $\left\{a_{k}\right\}$ be a sequence of distinct complex numbers. Then there is a function $f$ which is analytic in $D$ such that $\delta\left(a_{k}, f\right)=\delta_{k}$ and $\delta(b, f)=0$ for $b \in$ and $b \notin\left\{a_{k} \mid k=1,2, \ldots\right\} \quad($ Girnyk $[16])$.

There are some theorems which relate how a number of specific values are assumed when the function grows rapidly enough.

THEOREM 2.13. Let $f$ be a meromorphic function in $D$. Let $p(r)$ be the total number of roots in $\{z|| z \mid \leq r\}$ of the equations $f(z)=a_{k}, k=1,2, \ldots, q$ where the $a_{k}$ are $q$ distinct elements of $\mathbb{C} \cup\{\infty\}$ and $q \geq 3$. If

$$
\lim \inf \frac{(1-r) \log M(r, \varepsilon)}{-\log (1-r)}>\gamma
$$


where $\gamma$ is some finite positive number, then

$$
\lim _{r \rightarrow 1} \sup (1-r) p(r)>b
$$

where $b=\frac{1}{2} \gamma(q-1)$ or $\frac{1}{2} \gamma(q-2)$ according as the $a_{k}$ are all finite or not. If $f$ is an analytic function in $D$ and $(2.7)$ is replaced by

$$
\lim _{r \rightarrow 1} \sup \frac{(1-r) \log M(r, f)}{-\log (1-r)}>r
$$

the same conclusion is obtained, and the theorem is best posstble (Hayman [17]).

Relationships can be formulated between the defictencies of a function and the deficiencies of the derivatives of the function at zero.

THEOREM 2.14. Let $\mathrm{f}$ be a meromorphic function in $\mathrm{D}$ for which $\ell>0$ where $\ell$ is defined by $(2.2)$. Then

$$
\frac{1}{n+1} \sum_{a_{j} \neq \infty} \delta\left(a_{j}, f\right) \leq \delta\left(0, f^{(n)}\right), \text { for } n \geq 1 \text {. }
$$

If $f$ is an analytic function in $D$ for which $\ell>0$, then

(c.f. Sons) [4]).

$$
\sum_{j \neq \infty} \delta\left(a_{j}, f\right) \leq \delta\left(0, f^{(n)}\right), \text { for } n \geq 1
$$

Analogous results for functions meromorphic in the plane or for entire functions appear in $\mathrm{H}$. Wittich [18].

If the functions grow rapidly enough, some conclusions can also be made about the value distribution of the derivative functions or combinations of the function and its derivative functions. The next two theorems give important growth interrelations used to obtain such results.

THEOREM 2.15. Let $f$ be a meromorphic function in $D$ such that

$$
\lim _{r \rightarrow 1} \sup \frac{T(r, f)}{-\log (1-r)}=+\infty \text {. }
$$

Let $\nu$ be a positive integer, and define the function $\psi$ in $D$ by

$$
\psi(z)=\sum_{k=0}^{\nu} a_{k}(z) f^{(k)}(z)
$$

where $a_{i}(i=0,1, \ldots, \nu)$ is a meromorphic function in $D$ such that $T\left(r, a_{i}\right)=o(T(r, f)),(r+1)$. Then

$$
m(r, \psi / f)=o(T(r, f)),(r+1),
$$

and 


$$
T(r, \psi) \leq(\nu+1) T(r, f)+o(T(r, f)), \quad(r \rightarrow 1)
$$

THEOREY 2.16. Let $f$ be a meromorphic function in $D$, and assume (2.8) holds.

Let $\psi$ be as in THEOREY 2.13, and assume $\psi$ is not constant. Then

$T(r, f)<\bar{N}(r, f)+N(r, 1 / f)+\bar{N}\left(r, \frac{1}{\psi-\dot{1}}\right)-N_{0}\left(r, \frac{1}{\psi}\right)+o(T(r, f), \quad(r \rightarrow 1)$,

where in $\mathrm{N}_{0}\left(r, 1 / \psi^{\prime}\right)$ only zeros of $\psi^{\prime}$ not corresponding to the repeated roots of $\psi(z)=1$ are to be considered.

We proceed now to theorems about the values derivative functions assume. TFEORE:tS 2.15-2.19 are all analogues of theorems in Hayman [19] and/or Hayman [1].

THEOREM 2.17. Let $f$ be a meromorphic function in $D$ such that (2.8) holds. Suppose

$$
N(r, f)=o(T(r, f)),(r+1),
$$

and

$$
N(r, 1 / f)=o(T(r, f)),(r \rightarrow 1) \text {. }
$$

Let $\psi$ be defined as in THEOREM 2.15. Then $\psi$ is identically constant or $\psi$ assumes every finite complex value except possibly zero infinitely often. In fact, in the latter case, $\psi$ has no finite deficient values except possibly zero.

THEOREI 2.18. Let $f$ be a meromorphic function in $D$ such that (2.8) holds. Let $\nu$ be a positive integer Then

$$
\sum_{a \neq \infty} \Theta\left(a, f^{(v)}\right) \leq 1+\frac{1}{v+1}
$$

In particular, $\mathrm{f}^{(\mathrm{v})}$ assumes every finite value with at most one exception infinitely of ten.

THEOREM 2.19. Let $f$ be a meromorphic function in $D$ such that (2.8) holds. If $v$ is a positive integer, then

$$
T(r, f)=\left(2+\frac{1}{v}\right) N(r, 1 / f)+\left(2+\frac{2}{v}\right) \bar{N}\left(r, \frac{1}{f^{(v)_{-1}}}\right)+o(T(r, f)), \quad(r \rightarrow 1) .
$$

Consequently, either $f$ assumes every finite value infinitely often or $f(v)$ assumes every finite value except possibly zero infinitely often.

Meromorphic functions with order $\alpha<+\infty$ in $D$ have been considered as coefficients of certain differential equations in D. G. Valiron [20], s, Bank [21,22,23], and A.A. Gol'dberg $[24,25]$ have studied restrictions on the growth of the analytic or meromorphic functions in $D$ which are solutions of some differential equations for which the coefficients are analytic or meromorphic functions of finite order in $D$. 
Another gauge of values assumed is the Valiron defictency. If $f$ is a meromorphic function in $D$ and $a$ is in $C \cup\{\infty\}$ we define

$$
\Delta(a)=\Delta(a, f)=\lim _{r \rightarrow 1} \sup \frac{m(r, a)}{T(r, f)}=1-\lim _{r \rightarrow 1} \inf \frac{N(r, a)}{T(r, f)} .
$$

$\Delta(a)$ is called the Valiron deficiency of a. The next theorem gives some measure of the size of the set of Valiron deficient values.

THEOREM 2.20. Let $f$ be a meromorphic function in $D$. Assume

$$
\lim _{r \rightarrow 1} T(r, f)=+\infty \text {. }
$$

Then

$$
\lim _{r \rightarrow 1} \frac{N(r, a)}{T(r, f)}=1
$$

except for at most a set of a-values of vanishing inner capacity. Thus the Valiron deficiency $\Delta(a, f)$ is positive at most for a set of a-values of inner capacity zero, (Nevanlinna [6, p.280]).

From the definitions of Nevanlinna and Valiron deficiency it is clear that each Nevanlinna deficient value is also a Valiron deficient value. How are Borel exceptional values related to deficient values?

THEOREM 2.21. Let $f$ be a meromorphic function in $D$ which has finite order $\alpha$ and lower order $\ell$.

(i) If $a$ is a Borel exceptional value for $f$, then $\Delta(a, f)=1$.

(ii) If $a$ is a Borel exceptional value for $f$ and $\alpha=\ell$, then $\delta(a, f)=1$. (Sons [10]).

Another type of function behavior of special interest is the presence of radial limits, or asymptotic values in general. A function such as $f$ defined in $D$ by

$$
f(z)=\exp \left(\frac{1+z}{1-z}\right)
$$

has radial limits 1 and $+\infty$ as $|z| \rightarrow 1$ along the negative axis and positive axis respectively. However, we have the following theorem.

THEOREM 2.22. There exist functions meromorphic in $D$ which have no asymptotic values, finite or infinite (and hence no radial limits). Further, the Nevanlinna characterisitc of such functions may have arbitrarily slor growth. (Maclane [26]).

(0. Lehto and K. Virtanen [27] have shown that there even exist meromorphic functions $f$ in $D$ without asymptotic values which are also normal functions in $D-$ 
that is, they satisfy

$$
\frac{\left|f^{\prime}(z)\right|}{1+|f(z)|^{2}} \leq \frac{c}{1-|z|^{2}}
$$

for $z$ in $D$ and some constant C).

Meromorphic functions in $D$ which have no asymptotic values possess interesting value distribution properties.

THEOREM 2.23. A function meromorphic in $D$ which has no asymptotic values assumes every value infinitely often. Further, in every neighborhood of each point on $\{z|| z \mid=1\}$, the function assumes all except perhaps two values (c,f. Cartwright and Collingwood $[28,29])$.

The exceptional values in the theorem can occur even in such a way that the two values cluster only to one boundary point (Barth and Schneider [30]).

K. Barth [31] introduced the class $A_{m}$ of meromorphic functions in $D$ which have asymptotic values at a dense set on $\{z|| z \mid=1\}$. If $f$ is a meromorphic function in $D$ with order $\alpha<2$, then the following theorem gives a sufficient condition for f to be in $A_{m}$.

THEOREM 2,24. Let $f$ be a meromorphic function in $D$ which is not constant. If

$$
\int_{0}^{1}(1-r) T(r, f) d r<\infty,
$$

and for some a in $\mathbb{C} \cup\{\infty\}$

$$
N(r, a)=0(1), \quad(r \rightarrow 1)
$$

then $f$ is in $A_{m}$ (Barth [31]).

There are examples (Barth [31]) to show condition (2.9) cannot be relaxed to $\delta(a, f)=1$

Let $f$ be a non-constant meromorphic function in D. An asymptotic tract $\{D(\varepsilon), a\}$ assoclated with the finite complex number a is a set of non-vold domains $D(\varepsilon)$, one for each $\varepsilon>0$, such that

(1) $D(\varepsilon)$ is a component of the open set $\{z \mid z \in D u\{z|| f(z)-a \mid<\varepsilon\}\}$;

(11) $0<\varepsilon_{1}<\varepsilon_{2}$ Implies $\mathrm{D}\left(\varepsilon_{1}\right) \subset \mathrm{D}\left(\varepsilon_{2}\right)$; and 
(iii) $\underset{\varepsilon>0}{n} D(\varepsilon)=\phi$

If $a$ is replaced by to the only change is to replace $|f(z)-a|<\varepsilon$ in (i) by $|f(z)|>1 / \varepsilon$.

The set

$$
K=\underset{\varepsilon>0}{n} \overline{\mathrm{D}(\varepsilon)}
$$

is a non-void, connected, closed subset of $\{z|| z \mid=1\}, K$ is defined to be the end of the tract. If $\mathrm{K}$ is a point, then the tract will be termed a point-tract; if $\mathrm{K}$ is an arc, then the tract will be called an arc-tract. Barth [31] has given a suffir cient condition for the nonexistence of arc-tracts for functions in $A_{m}$.

THEOREM 2.25. Let $f$ be a meromorphic function in $D$ such that $f$ is in $A_{m}$. If there exist $a$ and $b$ in $\mathbb{C} \cup\{\infty\}$ with $a \neq b$ such that

$$
N(r, a)=0(1), \quad(r \rightarrow 1),
$$

and

$$
N(r, b)=0(1), \quad(r \rightarrow 1)
$$

then $f$ has no arc-tracts.

Another interesting question concerning $A_{m}$ is whether the derivative of a function in the class is again in the class.

THEOREM 2.26. Let $f$ be a meromorphic function in $D$. If

$$
N(r, \infty)=0(1), \quad(r \rightarrow 1),
$$

and

$$
\int_{0}^{1}(1-r) T(r, f) d r<\infty,
$$

then for each positive integer $n, f^{(n)}$ is in $A_{m}$ or $f^{(n)}$ is a constant (Barth and Schneider [32]), 


\section{SECTION THREE.}

We now consider only analytic functions in $D$ with growth measured by the maximum modulus of the function on circles centered at the origin. For such functions there are some types of theorems which are not available for meromorphic functions. Further, sometimes one can make added assertions for analytic functions. We define the M-order of such functions below.

Let $f$ be an analytic function in the unit disk $D$. Let

$$
\rho=\lim \sup _{r \rightarrow 1} \frac{\log ^{+} \log ^{+} M(r, f)}{-\log (1-r)} \text {. }
$$

where $M(x, f)$ is the maximum modulus of $f$ on $\{z|| z \mid=r\}$. We say $\rho$ is the M-order of $f$ and observe that $0 \leq \rho \leq+\infty$. We define the lower M-order $\lambda$ of $f$ by

$$
\lambda=\lim _{r \rightarrow 1} \inf \frac{\log ^{+} \log ^{+} M(r, f)}{-\log (1-r)} .
$$

According to M-order it can be shown that a function and its derivative function behave similarly. We have

THEOREM 3.1. Let $f$ be an analytic function in $D$ such that $f$ has M-order $\rho$ and lower M-order $\lambda$. If the derivative function $f^{\prime}$ has M-order $\rho^{\prime}$ and lower $M$ order $\lambda^{\prime}$, then $\rho=\rho^{\prime}$ and $\lambda=\lambda^{\prime}$ (c.f. Boas [33, p.13] and Sons [4]).

For an analytic function in $D$ it is possible to relate the growth of the function to the growth of the coefficients in its power series expansion about zero.

THEOREM 3.2. Let $f$ be an analytic function in $D$ with finite M-order $\rho$. If

$$
f(z)=\sum_{n=0}^{\infty} a_{n} z^{n}, \quad(z \in D)
$$

then

$$
\frac{\rho}{\rho+1}=\lim \sup _{n \rightarrow \infty} \frac{\log ^{+} \log ^{+}\left|a_{n}\right|}{\log n} \text { and } \rho=\lim \sup _{n \rightarrow \infty} \frac{\log ^{+} \log ^{+}\left|a_{n}\right|}{\log n-\log ^{+} \log ^{+}\left|a_{n}\right|} \text {, }
$$

(MacLane [34, p.38].

THEOREM 3.3. Let $f$ be an analytic function in $D$ with finite lower M-order $\lambda$. If $f(z)$ has the form (3.3), then

(i) $\frac{\lambda}{1+\lambda} \geq \lim \inf _{n \rightarrow \infty} \frac{\log ^{+} \log ^{+}\left|a_{n}\right|}{\log n}$. 
where equality holds if there is an $n_{0}$ such that $\left|a_{n} / a_{n+1}\right|$ is nondecreasing for $\mathbf{n}>\mathbf{n}_{\mathbf{0}}$;

$$
\begin{aligned}
& \text { (ii) } 1+\lambda \geq \lim _{n \rightarrow \infty} \inf _{n \rightarrow \infty} \frac{\log (n-1)}{\log \left(1 /\left(\log ^{t}\left|a_{n} / a_{n-1}\right|\right)\right)} \text {; } \\
& \text { (iii) } 1+\lambda \geq \lim _{n \rightarrow \infty} \operatorname{linf}_{n \rightarrow \infty} \frac{\log (n-1)}{\log n-\log ^{+} \log ^{+}\left|a_{n}\right|} \text {, }
\end{aligned}
$$

(Kapoor [35] and Kapoor and Juneja [36]).

A theorem of a slightly different nature comes by considering the maximum term and central index in the power series expansion.

THEOREM 3.4. Let $f$ be an analytic function in $D$ with M-order $\rho$ such that $0<\rho<\infty$ and lower M-order $\lambda$. Suppose $f(z)$ has the form (3.3). Let $\mu(r)$ be the maximum term in (3.3) for $\{z|| z \mid=r\}$ and $v(r)$ be its central index. Then

$$
\begin{aligned}
& \text { (i) } \lim _{r \rightarrow 1} \sup _{\text {inf }_{\}}} \frac{\log ^{+} \log ^{+} \mu(r)}{-\log (1-r)}=\left\{\begin{array}{l}
\rho \\
\lambda
\end{array} ;\right. \\
& \text { (ii) } 1 \text { im sup } \frac{\log v(r)}{-\log (1-r)}=1+\rho ; \\
& \text { (iii) } \lim _{r \rightarrow 1} \frac{\log v(r)}{-\log (1-r)} \leq 1+\lambda,
\end{aligned}
$$$$
\text { and }
$$

The function defined in $D$ by

$$
\sum_{j=0}^{\infty} e^{k_{j}\left(k_{j}\right)^{2}}
$$

where $k_{0}$ is 2 and $k_{j+1}=\left(k_{j}\right)^{2}$ shows equality need not hold in the last statement.

A well known inequality relates the Nevanlinna characteristic of a function to the maximum modulus. If $f$ is an analytic function in $D$, then

$$
T(r, f) \leq \log ^{+} M(r, f) \leq \frac{R+r}{R-r} T(R, f), \quad(0 \leq r<R<1) .
$$

THEOREM 3.5 is a direct consequence of this inequality.

THEOREM 3.5. Let $f$ be an analytic function in $D$ with finite M-order $\rho$. If $\alpha$ is the order of $f$ in $D$ as defined by (2.1), then $\alpha \leq \rho \leq \alpha+1$. Using the notation of THEOREM 3.5 we observe that the functions $f_{k}$ defined in 
D. for $k>1$ by

$$
f_{k}(z)=\exp \left((1-z)^{-k}\right)
$$

have $M$-order $\rho=k$ and order $\alpha=k-1$. To see that other possibilities allowed by the theorem can be assumed, we give two theorems.

THEOREM 3.6. Let $\psi$ be a convex function defined for $r$ in $0 \leq r<1$ and satisfying

$$
\lim _{r \rightarrow 1} \frac{\psi(r)}{-\log (1-r)}=+\infty \text { and } \lim _{r \rightarrow 1} \sup \frac{\log \psi(r)}{-\log (1-r)}<+\infty
$$

Then there exists a function $f$ analytic in $D$ such that

$$
T(r, f) \sim N(r, 1 / f) \sim \log M(r, f) \sim \psi(r), \quad(r \rightarrow 1), \quad \text { (Shea [38]). }
$$

THEOREM 3.7. Let $\phi$ and $\psi$ be a non-negative, increasing functions which are defined for $r$ in $0 \leq r<1$, which are convex with respect to $\log r$ in $0 \leq r<1$, and which satisfy

$$
\begin{gathered}
\lim _{r \rightarrow 1} \frac{\phi(r)}{-\log (1-r)}=+\infty, \lim _{r \rightarrow 1} \frac{\psi(r)}{-\log (1-r)}=+\infty, \\
\psi(r)=o(\phi(r)), \\
\phi(r)(1-r)+\int_{0}^{r} \phi(t) d t=o(\psi(r)), \quad(r \rightarrow 1),
\end{gathered}
$$

and $\phi^{\prime}(r)(1-r)$ is monotonically increasing for $r$ in the interval $(0,1)$. Then there is a function $f$ analytic in $D$ such that

$$
\log M(r, f) \sim \phi(r) \text { and } T(r, f) \sim \psi(r), \quad(r \rightarrow 1)
$$

(LInden [39]).

Turning to a consideration of values assumed by our functions, we see:

THEOREM 3.8. If $f$ is an analytic function in $D$ with M-order $\rho>1$, then THEOREM 2.2 applies and $f$ omits at most one value. If $f$ is an analytic function in $D$ with $M$-order $\rho=1$, then $f$ may omit infinitely many values.

The first part of THEOREM 3.8 follows from setting $R=r+\frac{1}{2}(1-r)$ in inequality (3.4) to get the implication

$$
\lim _{r \rightarrow 1} \sup \frac{\log T(r)}{-\log (1-r)}>0
$$


To see the second part we note that $g$ defined in $D$ by

$$
g(z)=\exp \left(\frac{1+z}{1-z}\right)
$$

has M-order $\rho=1$ and $|g(z)|>1$ for $z$ in $D$.

Translating THEOREM 2.6 for an analytic function $f$ in $D$ shows that such an $f$ can have at most one Borel exceptional value. In fact, $f$ defined by $f(z)=$

$\exp \left((1-z)^{-3 / 2}\right)$ has zero as a Borel exceptional value and 1 -order $\rho=3 / 2$. This function also shows that the M-order of a function analytic in $D$ with a Borel exceptional value need not be an integer. Indeed it can be shown that analytic functions in $D$ with a Borel exceptional value need not have regular growth according to either order or M-order.

THEOREM 3.9. There exist analytic functions $f$ in $D$ which have order $\alpha$, lower order $\ell, M$-order $\rho$, and lower M-order $\lambda$ such that

$$
f(z)=\exp (g(z))
$$

for $z$ in $D$ where $g$ is an analytic function in $D$, and both $\lambda \neq \rho$ and $l \neq \alpha$ (Sons [10]).

By using a canonical product which is essentially that of $M$. Tsuji in SECTION TWO we obtain a factorization of functions analytic in $D$ when the M-order is large enough.

THEOREM 3.10. Let $f$ be an analytic function in $D$ with M-order $\rho$ where $1 \leq \rho<\infty$, and let $\left\{a_{n}\right\}$ be the zeros of $f$ in $D$. If $s$ is an integer not less than [o], the function $p$ defined by

$$
p(z)=\prod_{n=1}^{\infty} \frac{a_{n}\left(a_{n}^{-z}\right)}{\left(1-z \bar{a}_{n}\right)} \exp \sum_{k=1}^{s+1} \frac{1}{k}\left(\frac{1-\left|a_{n}\right|^{2}}{1-z_{n}}\right)^{k},
$$

is analytic in $D$ and of M-order at most $\rho$ (Linden [40]).

THEOREM 3.11. Let $f$ be an analytic function in $D$ with finite M-order $\rho$ where $\rho \geq 1$. Then

$$
f(z)=p(z) q(z),
$$

where $p$ is of the form (3.5) and formed using the zeros of $f$, and both $p$ and $q$ 
are analytic and of M-order at most $\rho$ in D. (Linden [40]),

Making additional hypotheses concerning the zeros of $f$, we obtain a theorem concerning functions of smaller Morder growth,

THEOREN 3.12, Let $f$ be an analytic function in $D$ which has finite Forder $\rho$. Let $\left\{a_{n}\right\}$ be the zeros of $f$ in $D$, and define $\tilde{n}(r, \theta, f)$ to be the number of these zeros satisfying

$$
r \leq\left|a_{n}\right|<\frac{1}{2}(1+r) \text { and }\left|\theta-\arg a_{n}\right| \leq \pi(1-r) .
$$

Set

$$
n(r, f)=\max _{0 \leq \theta<2 \pi} \tilde{n}(r, \theta, f) \text { and } \gamma=\lim _{r \rightarrow 1} \sup \frac{\log ^{+} \eta(r, f)}{-\log (1-r)} .
$$

Then

$$
f(z)=p(z) q(z), \quad(z \in D),
$$

where $p(z)$ is of the form (3.5) with $s=[\gamma]$ and both $p$ and $q$ are analytic functions in $D$ with M-order at most $\max (\rho, \gamma)$ (Linden [40]).

Using Jensen's formula it can be seen that if $f$ is an analytic function in $D$ and $|f(0)|=1$, then

$$
\mathrm{n}(\mathrm{r}, \mathrm{f})<\frac{\log \mathrm{M}(\mathrm{R}, \mathrm{f})}{\log (\mathrm{R} / \mathrm{r})}, 0<\mathrm{r}<\mathrm{R}<1 .
$$

If $f$ has finite M-order $\rho$, then taking $R=\frac{1}{2}(1+r)$ we find for $\varepsilon>0$ there is an $\mathbf{r}_{0}$ such that

$$
n(r, f)<(1-r)^{-1-\alpha-\varepsilon}
$$

for $r_{0} \leq r<1$. If a function has nearly all the zeros this allows, the zeros will need to be fairly uniformly distributed near $\{z|| z \mid=1\}$ as THEOREM 3.13 shows.

THEOREM 3.13. Let $f$ be an analytic function which has M-order $\rho$ in $D$ where $1 \leq \rho<\infty$. Then for each positive $\varepsilon$ and any $\theta \quad(0 \leq \theta<2 \pi)$, there is a number $r_{0}$ such that the number of zeros of $f$ in

$$
r \leq|z| \leq \frac{1}{2}(1+r),|\theta-\arg z| \leq \pi(1-r),
$$

is at most $(1-r)^{-\rho-\varepsilon}$ for $r_{0} \leq r<1$ (Linden [40]).

For smaller M-order we have:

THEOREM 3,14, Let $f$ be an analytic function in $D$ which has M-order $\rho<1$. Suppose $\rho<n$. Then there is a constant A such that the number of zeros of $f$ in any region

$$
\left\{z|| \sin \frac{1}{2}(\theta-\arg z)\left|<(1-r)^{n}, \quad r \leq\right| z \mid<\frac{1}{2}(1+r)\right\}
$$


is less than $\mathrm{A} /(1-\mathrm{r})$ whenever $0 \leq \mathrm{r}<1$ and $0 \leq \theta<2 \pi$ (Linden [41]).

If restrictions are made regarding the location of the zeros of a function, the bound in (3.6) can be made better. THEOREM 3.15 gives an example.

THEOREM 3.15. Let $f$ be an analytic function in $D$ which has finite M-order $\rho$. Suppose that the zeros of $f$ all lie on a finite number of radil of $\{z|| z \mid=1\}$. Then if $\varepsilon>0$ and $q=\max (\rho+\varepsilon, 1)$, there is a constant $C$ such that

$$
\mathrm{n}(\mathrm{r}, \mathrm{f})<\mathrm{C}(1-\mathrm{r})^{-\mathrm{q}}
$$

whenever $0 \leq \mathrm{r}<1$ (Linden [41]).

In general for functions with order less than one, it is not possible to improve (3.6) much. In particular, if we let

$$
a_{k}=1-\frac{1}{k(\log k+2)^{2}}, \quad(k=1,2, \ldots)
$$

and let $B$ be defined in $D$ by

$$
B(z)=\prod_{k=1}^{\infty} \frac{\bar{a}_{k}}{\mid a_{k}}\left(\frac{a_{k}-z}{1-z \bar{a}_{k}}\right),
$$

then $B$ is an analytic function in $D$ which has M-order zero and

$$
n(r, 1 / B) \sim \frac{1}{(1-r)\{\log (1-r)\}^{2}} \quad(r \rightarrow 1)
$$

(c.f. Linden [41]).

Turning our attention to values assumed by the derivative function, we shall give two theorems.

THEOREM 3.16. If $f$ is an anlaytic function in $D$ with

$$
\underset{r \rightarrow 1}{\lim \sup }(1-r) \log M(r, f)=+\infty,
$$

then either $f$ assumes every complex number infinitely often, or every derivative of f assumes every complex number except possibly zero infinitely often (c.f. Hayman [17] and [19]).

The exceptional position of zero in the above theorem cannot be eliminated by assuming a stronger growth condition. Functions $f$ defined in $D$ by 


$$
f(z)=\exp \left(\int \exp g(z) d z\right)
$$

where $g$ is an arbitrary function which is analytic in $D$ satisfy $f(z) \neq 0$ and $f^{\prime}(z) \neq 0$ for any $z$ in $D$. So for the first derivative one cannot strengthen THEOREM 3.16. However, one can prove:

THEOREM 3.17. If $f$ is an analytic function in $D$ with

$$
\lim _{r \rightarrow 1} \sup (1-r) \log \log M(r, f)=+\infty
$$

then either $f$ or $f^{\prime \prime}$ assumes every complex number infinitely of ten (Hayman [19]).

Suppose $f$ is an analytic function defined in $D$ by

$$
f(z)=\exp \left(\frac{\gamma}{1-z}\right)
$$

where $\gamma$ is a positive number. If $E$ is a fixed bounded set and $\nu$ a fixed positive integer, then

$$
\lim _{r \rightarrow 1}(1-r) \log M(r, f)=r
$$

and if $\gamma$ is sufficiently large, $f(z), f^{\prime}(z), \ldots, f^{(v)}(z)$ for $z$ in $D$ assume no value in E. Thus the growth hypothesis in THEOREM 3.16 cannot be sharpened (Hayman [19]).

To see the growth hypothesis in THEOREM 3.17 cannot be sharpened, we define f and $g$ in $D$ by

$$
f(z)=\exp \left(\int_{0}^{z} g(\zeta) d \zeta\right)
$$

where

$$
g(z)=\exp \left(a\left(\frac{1}{1-z}+\frac{1}{(1-z)^{1 / 2}}\right)\right)
$$

and $a$ is a positive constant. Clearly $f$ is an analytic function in $D$ wth $f(z) \neq 0$ and $f^{\prime}(z) \neq 0$ for $z$ in $D$. For all sufficiently large a, one can show also $f^{\prime \prime}(z) \neq 0$ for $z$ in $D$ (Hayman [19]).

The situation concerning asymptotic values for functions analytic in $D$ is different from that for meromorphic functions. We give three theorems concerning the exis tence of such values. 
THEOREM 3.18. Let $f$ be an analytic function in $D$. Then $f$ has at least one asymptotic value (MacLane [34, p.7] and Nevanlinna [6, p.292]).

THEOREM 3.19. Let $f$ be an analytic function in $D$. Then $f$ need not have a radial limit along any ray (Bagemihl, Erd68, and Seidel [42, Th.7] and MacLane [43]) .

THEOREM 3.20. Let $f$ be an unbounded analytic function in $D$. Then $f$ need not have $\infty$ as an asymptotic value (MacLane [34, p.71]).

At a point on the boundary of $D$ an analytic function may have more than one asymtotic value, but it is possible to determine the size of the set of such points.

THEOREM 3.21. Let $f$ be an analytic function in $D$. Then the set of points $E$ on $\{z|| z \mid=1\}$ at which $f$ has more than one asymptotic value is at most countable (Bagemihl [44] and Heins [45]).

G. R. MacLane introduced the class $A$ of non-constant analytic functions in $D$ to be those functions which have asymptotic values at a dense set on $\{z|| z \mid=1\}$. THEOREM 3.22 gives the limitations on the growth of such functions.

THEOREM 3.22. Let $f$ be a non-constant analytic function in $D$. If

$$
\int_{0}^{1} \log ^{+} \log ^{+} M(r, f) d r<+\infty,
$$

then $f \in A$. In particular, if $f$ has fintte M-order, then $f \in A$. Further, for $\varepsilon>0$. there exists a non-constant function $f$ which is analytic in $D$ such that f \& $A$ and for some number $r_{0}$ with $0<r_{0}<1$,

$$
M(r, f)<\exp \exp \frac{\varepsilon}{(1-r)(-\log (1-r))},\left(r_{0}<r<1\right), \quad \text { (Hornblower [46]). }
$$

(G. R. MacLane [47] and P. J. Rippon [48] both give different discussions of THEOREM 3.22).

MacLane [47] also introduces the classes $B$ and $L$ of non-constant analytic functions in D. $B$ is the class such that there is a set of Jordan arcs $\Gamma$ in $D$, each ending at a point of $\{z|| z \mid=1\}$ and such that the end points are dense on $\{z|| z \mid=1\}$ and such that on each $\Gamma$ either $f$ tends to infinity or $f$ is bounded. To define $L$ we first need the notion of a set ending at points of the unit circle.

Let $S$ be a subset of $D$. For each $r$ with $0<r<1$, label the components of 
$S \cap\{z|r<| z \mid<1\}$ by $S_{k}(r)$ where $k$ is a non-negative integer. Let $d_{k}(r)$ be the diameter of $S_{k}(r)$, and set

$$
d(r)=\sup _{k} d_{k}(r)
$$

with $d(r) \equiv 0$ if there are no components $s_{k}(r)$. We say $s$ ends at points of $\{z|| z \mid=1\}$ if and only if $d(r)$ decreases to zero as $r$ approaches one.

$L$ is then the class of non-constant analytic functions $f$ in $D$ for which every level set $\{z|| f(z) \mid=\lambda\}, \lambda \geq 0$, ends at points of $\{z|| z \mid=1\}$.

MacLane's startling result is that $A=B=L$, so a non-constant analytic function $f$ in $D$ with finite M-order by THEOREM 3.22 is also in $B$ and in $L$.

(In [31] K.F. Barth defined the corresponding classes $B_{m}$ and $L_{m}$ of nonconstant meromorphic functions in $D$, but the classes turn out to be distinct from each other and from $A_{m}$ as defined in SECTION TWO).

Actually, a function which is analytic in $D$ may have infinitely many asymptotic values at a point on $\{z|| z \mid=1\}$. Such a function was noted in [34] by considering the entire function $F$ of $W$. Grosz [49] for which every complex number is an asymptotic value. If the plane is slit along one path on which $F \rightarrow \infty$, then the slit plane can be mapped onto $D$. The function $f$ defined in $D$ by means of the composition of the map and $F$ is a function in $A$ which has every complex number as an asymptotic value at one point $z_{0}$ with $\left|z_{0}\right|=1$.

THEOREM 3.23. tells us that the number of asymptotic tracts at a point for a function analytic in $D$ is related to the order of growth of the function.

THEOREM 3.23. Let $f$ be a non-constant analytic function in $D$. Suppose there is a number $\gamma$ with $\gamma \geq 0$ such that

$$
\int_{0}^{1}(1-r)^{r} \log M(r, f) d r<\infty \text {. }
$$

Let $z_{0}$ be a complex number such that $\left|z_{0}\right|=1$, and let $n_{f} n_{\infty}$ ) denote the number of distinct asymptotic tracts of $f$ associated with $z_{0}$ for which the asymptotic values are finite (infinite). Then

$$
n_{f} \leq[\gamma+1] \text { and } n_{\infty} \leq[\gamma+2]
$$


where [ ] is the usual greatest integer function. (Drasin [50] and liacLane [34, p.54]).

THEOREM 3.23 is best possible. To see this is true for $\gamma=0$, consider $f$ defined in $D$ by

$$
f(z)=\frac{1+z}{1-z} \exp \left(\frac{1+z}{1-z}\right)
$$

for which $n_{f}=1$ and $n_{\infty}=2$ at $z_{0}=1$. To see the result is best possible when $0<\gamma \leq 1$, we consider $\mathrm{f}$ defined in $\mathrm{D}$ by

$$
f(z)=\left(\frac{1-z}{1+z}\right)^{1+\delta} \sinh \left(\frac{1+z}{1-z}\right)^{1+\delta}
$$

where $0<\delta<\gamma \leq 1$ for which $n_{f}=2$ and $n_{\infty}=3$ at $z_{0}=1$ (MacLane [34, p.77781).

When the number of tracts for $\infty$ is restricted for an analytic function in $D$, it is possible to say more about the set where the function has finite asymptotic values.

THEOREM 3.24. Let $f$ be an analytic function in $D$. If $f$ has only finitely many tracts for $\infty$ and the ends of the arc tracts of $f$. for $\infty$ do not cover $\{z|| z \mid=1\}$, then the Lebesgue measure of the set $A$ is positive where $A$ is the set of $\zeta$ with $|\zeta|=1$ for which there is a complex number a such that $f$ has the asymptotic value a at $\zeta$ (McMillan [51]).

Another growth behavior of interest is how the minimum modulus of a function on a circle about the origin acts.

THEOREM 3.25. Let $f$ be an analytic function in $D$ with finite M-order $\rho$ where $\rho>1$. Then there exists a constant $K$ such that

$$
\log L(r, f)>-K(\log M(r, f))(\log \log M(r, f))
$$

for a sequence of numbers $r$ increasing to one where

(Linden $[52,53])$.

$$
L(r, f)=\min _{|z|=r}|f(z)|
$$

The above theorem is best possible (c.f. Linden [52,53]). For functions of lesser growtin there is THEOREM 3.26 . 
THEOREM 3.26. Let $f$ be a non-constant analytic function in $D$ which has order $\rho$ where $\rho<1$. Then there exist positive constants $K$ and $C$ and a number $s_{0}$ with $0<s_{0}<1$ such that if $s$ satisfies $s_{0}<s<1$, the interval $\left(s, \frac{1}{2}(1+s)\right)$ contains a set of values $r$ of measure at least $\mathrm{C}(1-\mathrm{s})$ for which

$$
\log L(r, f)>-\frac{K}{1-r} \log \left(\frac{1}{1-r}\right)
$$

where $L(r, f)$ is defined by (3.7). The constant $C$ need not be less than $\frac{1}{4}$ (Linden [54]).

Finally, we shall look at the subset of $D$ in which a given function has its maximum modulus greater than one and observe how this set intersects $\{z|| z \mid=r\}$ for $0<r<1$. First we introduce some notation.

Let $f$ be an analytic function in $D$, and let

$$
\tilde{D}=\{z|| f(z) \mid>1 \text { and } z \in D\} \text {. }
$$

For $0<r<1$ let $A_{k}(r)$ be the arcs of $\{z|| z \mid=r\}$ which are contained in $\tilde{D}$, and let $r \theta_{k}(r)$ be their lengths. For $0<r<1$, if $\{z|| z \mid=r\}$ lies wholly in $\tilde{D}$, define $\theta(r)=+\infty$; otherwise set $\theta(r)=\max _{k} \theta_{k}(r)$. The following two theorems follow from a line of reasoning used by $K$. Arima in [55].

THEOREM 3.27. Let $f$ be an analytic function in $D$ which has finite M-order $\rho>0$ and lower M-order $\lambda$.

(i) If $\lambda=0$, then

$$
\underset{r \rightarrow 1}{\lim \sup } \frac{\theta(r)}{1-r} \geq 2 \pi
$$

(ii) If there exist a number $r_{0}$ such that $0<r_{0}<1$ and

$$
0<\theta(r) \leq \frac{2 \pi(1-r)}{1+2 p}
$$

for $r_{0}<r<1$, then $\rho=\lambda$.

THEOREM 3.28. Let $f$ be an analytic function in $D$ which has finite positive order $\alpha$ defined by (1.1). If there exists a number $r_{0}$ such that

$$
0<\theta(r) \leq \frac{2 \pi}{2+2 \gamma}(1-r)
$$


for $r_{0}<r<1$, then $\gamma \leq \alpha$.

Some subclasses of finite M-order have recelved special study.

If $f$ is an analytic function in $D, f$ is said to be in $B^{-n}$ if and only if

$$
|f(z)| \leq \exp \left(C(1-|z|)^{-n}\right), \quad(z \in D),
$$

where $C$ is a positive constant and $0<n<\infty$. If $f$ is an analytic function in $D$, $f$ is said to be in ${ }_{B}^{p}(0<p<\infty)$ if and only if

$$
\int_{|z|<1}\left(\log ^{+}|f(z)|\right)^{p} d x d y<\infty,
$$

E. Beller [56] points out that these classes are related by the following inclusions:

$$
B^{-\mathrm{n}} \subset \mathrm{B}^{(1 / \mathrm{n})}+\varepsilon,
$$

for all $\varepsilon>0$ and $0<\mathrm{n}<\infty$;

$$
{ }_{B}{ }^{p} \subset 3^{-2 / p}, \quad(1 \leq p<\infty) \text {. }
$$

Beller $[56,57]$ has proved the following theorem concerning the zeros of functions in these classes.

THEOREM 3.29. (i) If $f$ is an analytic function in $D$ which is in $B^{-n}$ where $0<\mathrm{n}<\infty$ and $\left\{\mathrm{a}_{\mathrm{k}}\right\}$ are the zeros of $\mathrm{f}$ in $\mathrm{D}$, then for $\varepsilon>0$,

$$
\sum_{k=1}^{\infty}\left(1-\left|a_{k}\right|\right)^{n+1+\varepsilon}<\infty .
$$

(i1) If $f$ is an analytic function in $D$ which is in ${ }_{B}{ }^{p}$, where $1 \leq p<\infty$, and the $\left\{a_{k}\right\}$ are the zeros of $f$ in $D$, then for $\varepsilon>0$,

$$
\sum_{k=1}^{\infty}\left(1-\left|a_{k}\right|\right)^{1+1 / p+\varepsilon}<\infty .
$$

For $B^{1}$ we get further information by taking $\alpha=0$ in the theorem of $A$. Heilper [58] which follows.

THEOREM 3.30. If $\mathrm{f}$ is an analytic function in $\mathrm{D}$ with

$$
\iint_{|z|<1}\left(\log ^{+}|f(z)|\right)(1-|z|)^{\alpha} d x d y<\infty
$$


for some real number $\alpha \geq 0$ and $\left\{a_{k}\right\}$ are the zeros of $f$, then

$$
\sum_{k=1}^{\infty}\left(1-\left|a_{k}\right|\right)^{2+\alpha}<\infty \text {. }
$$

If $0 \leq \alpha \leq 1$, the condition is sufficient.

H.S. Shapiro and A,L. Shields [59] showed that for some $n$, if $f$ is a function in $B^{-n}$ and $\left\{a_{k}\right\}$ are the zeros of $f$ on a single radius of the unit circle, then $\sum_{k=1}^{\infty}\left(1-\left|a_{k}\right|\right)<\infty$. Their theorem was extended by W.K. Hayman and B. Korenblum [60]: THEOREI 3.31. If $\phi$ is a positive, continuous non-decreasing function of $r$ on $[0,1)$ such that $\phi(r)$ approaches infinity as $r$ approaches one, and if $f$ is an analytic function in $D$ for which

$$
\log |f(z)| \leq \phi(|z|), \quad(z \in D)
$$

then those zeros $\left\{a_{k}\right\}$ of $f$ which lie on a single radius of the unit circle satisfy

$$
\sum_{k=1}^{\infty}\left(1-\left|a_{k}\right|\right)<\infty
$$

if and only if

$$
\int_{0}^{1} \sqrt{\frac{\phi(r)}{1-r}} d r<\infty
$$

C.N. Linden [61] has found additional conditions on $\phi$ in the previous theorem besides (3.9) to conclude that (3.8) holds for the zeros of $f$ in certain tangential regions in D. Bruce Hanson [62] has shown further relations between the growth of an analytic function in $D$ and the behavior of its zeros which lie on a single radius of the unit circle and also has some interesting examples concerning their best-possible nature. He shows:

THEOREM 3.32. Let $f$ be an analytic function in $D$, and let $\left\{a_{k}\right\}$ be the zeros of $f$ which lie on a single radius of the unit circle.

(i) If $0<\lambda<\infty$ and if

$$
\int_{0}^{1}\left(\log ^{+} M(r, f)\right)(1-r)^{\lambda} d r<\infty
$$

then

$$
\sum_{k=1}^{\infty}\left(1-\left|a_{k}\right|\right)^{\lambda+1}<\infty
$$


(1i) If

$$
\int_{0}^{1} \log ^{+} M(r, f) d r<\infty,
$$

then

$$
\sum_{k=1}^{\infty} \frac{1-\left|a_{k}\right|}{-\log \left(1-\left|a_{k}\right|\right)}<\infty \text {. }
$$

Another interesting class of functions in $D$ is those defined by gap power series. If the gaps are large and regular, the conclusions on value distribution are especially nice even for functions which grow slowly:

THEOREM 3.33. Let $f$ be a non-constant analytic function in $D$ defined by the power series representation

$$
f(z)=\sum_{k=0}^{\infty} c_{k} z^{n k}
$$

where the sequence $\left\{n_{k}\right\}$ satisfies

$$
\frac{n_{k+1}}{n_{k}} \geq q>1, \quad(k=1,2,3, \ldots),
$$

for some real number $q$.

(i) If

$$
\sum_{k=1}^{\infty}\left|c_{k}\right|=\infty,
$$

then $f$ assumes every complex number infinitely often. In fact, $f$ assumes every complex number infinitely often in every sector in $D$ of the form

$$
\{z|\alpha<\arg z<\beta, 0<| z \mid<1\},
$$

where $\alpha$ and $\beta$ are in $[-2 \pi, 2 \pi]$ (Murat [63]).

(ii) If

$$
\underset{k \rightarrow \infty}{\lim \sup }\left|c_{k}\right|>0
$$

then

$$
\delta(a, f)=0
$$

for all a in (Pfurai [64]).

THEOREM 3.34. Let $f$ be a non-constant analytic function in $D$ defined by the power series representation (3.10) where the sequence $\left\{n_{k}\right\}$ satisfies (3.11). Then (i) $f$ is in $A$;

(ii) if

$$
\underset{k \rightarrow \infty}{\lim \sup _{k}}\left|c_{k}\right|>0,
$$

then $f$ has no finite asymptotic values (Mural [65]). 
For smaller gaps in the series representation for the function, there are also value distribution results -- ones relating the gap size and the growth of the function.

THEOREM 3.35. Let $\mathrm{f}$ be a non-constant analytic function in $\mathrm{D}$ which has $M-$ order $\rho$ and is defined by the power series representation $(3,10)$ where the sequence $\left\{n_{k}\right\}$ satisfies

$$
\lim _{k \rightarrow \infty} \inf \frac{\log \left(n_{k+1}-n_{k}\right)}{\log n_{k+1}}>\frac{1}{2}\left(\frac{2+p}{1+p}\right)
$$

Then

(i) for each complex number $a, \delta(a, f)=0$;

$$
\underset{r \rightarrow 1}{\lim \sup }(L(r, f) / M(r, f))=1,
$$

where $L(r, f)$ is defined by $(3.7)$;

and

(iii) $f$ has no finite asymptotic values (Wiman [66]).

In [67] the conclusion (i) of THEOREM 3.35 is obtained for some functions with less restrictive gaps than (3.12). A result where the density of the sequence $\left\{n_{k}\right\}$ and the growth of the function is linked follows.

THEOREM 3.36. Let $f$ be a non-constant analytic function in $D$ defined by the power series representation (3.10). Let $\psi(t)$ be the number of $n_{k}$ not greater than $t$, and suppose that, for some fixed $B(0<\beta<1)$,

$$
\psi(t)=0\left(t^{1-\beta}\right), \quad(t \rightarrow \infty)
$$

If the M-order $\rho$ of $f$ satisfies

$$
\rho>\frac{1-\beta}{\beta} \text {, }
$$

then

(i) $\delta(a, f)=0$ for every complex number a, (Nicholls and Sons [68]);

and

(ii) $f$ has no finite asymptotic values (Sons [69]).

The best possible nature of $(3,13)$ to obtain (ii) is sinown for $n_{k}$ of the form $\mathrm{k}^{\mathrm{p}}$, where $\mathrm{p}$ is an integer not less than 2, by examples in [70] 
4. SECTION FOUR,

In this section we give some theorems concerning functions meromorphic in D with growth more restrictive than that in SECTION TWO. Define $\tilde{\alpha}$ and $\tilde{\ell}$ by

$$
\underset{r \rightarrow 1}{\lim \sup _{r \rightarrow 1}} \frac{T\left(r_{2} f\right)}{-\log (1-r)}=\tilde{\alpha}
$$

and

$$
\lim _{r \rightarrow 1} \inf \frac{T(r, f)}{-\log (1-r)}=\tilde{l}
$$

where $T(r, f)$ is the Nevanlinna characteristic of $f$ at $r$. Then $0 \leq \tilde{l} \leq \tilde{\alpha} \leq+\infty$.

It is possible to obtain some restriction on the growth of the derivative of a function in this class in terms of the growth of the function.

THEOREM 4.1. Let $f$ be a meromorphic function in $D$ with $\tilde{\alpha}$ defined by (4.1) and $\tilde{\alpha}<+\infty$. Then

$$
\lim _{r \rightarrow 1} \frac{T\left(r, f^{\prime}\right)}{-\log (1-r)} \leq 2(\tilde{\alpha}+1)
$$

If $f$ is in fact analytic in $D$, then

$$
\lim _{r \rightarrow 1} \sup _{r\left(r, f^{\prime}\right)} \frac{T}{-\log (1-r)} \leq \tilde{\alpha}+2
$$

The growth of the function places some restriction on the number of a-values and placement in the disc where $f$ may assume a-values.

THEOREM 4.2. Let $f$ be a meromorphic function in $D$ with $\tilde{\alpha}$ defined by (4.1) and $\tilde{\alpha}<+\infty$. Let $\left\{{ }^{a} k\right\}$ be the set of a-values for $f$ where a is in $\mathbb{C} U\{$. Then

(i) for each $\epsilon>0, \sum_{k}\left(1-\left|a_{k}\right|\right)^{1+\epsilon}<\infty$;

(ii) the product

$$
P(z)=\pi\left(1-\frac{1-\left|a_{n}\right|^{2}}{1-\bar{a}_{n} z}\right) \exp \left(\frac{1-\left|a_{n}\right|^{2}}{1-\bar{a}_{n} z}\right),
$$

converges for each $z$ in $D$;

(iii) for each $\varepsilon>0$ there exists a constant $K$ such that

$$
\lim \sup _{\substack{r \rightarrow 1 \\ r \notin C_{n}}} \frac{(1-r)^{1+\varepsilon} \log ^{+}(1 /|P(r)|)}{-\log (1-r)} \leq K \text {. }
$$


where $c_{n}=\left\{z|| z-a_{n} \mid \leq\left(1-\left|a_{n}\right|^{2}\right)^{4}\right\}$ for $n=1,2, \ldots$ (Tsuj1 [3, p. 204]).

A restriction on the possible number of zeros (or a-values) is given below. THEOREM 4.3. Let $f$ be a meromorphic function in $D$ for which $0<\tilde{\alpha}<+\infty$ with $\tilde{\alpha}$ defined by $(4,1)$. Then

$$
\underset{r \rightarrow 1}{\lim \sup _{\mathrm{p}}} \frac{(1-r) n(r)}{-\log (1-r)} \leq 2 \tilde{\alpha} . \quad \text { (Sons [4]). }
$$

We now consider the number of values a function in our class can omit and number of times values are assumed relative to the growth of the function.

Let $f$ be a meromorphic function in $D$, and define $\tilde{\mu}_{a}$ for each $a$ in $c$ by

$$
\underset{r \rightarrow 1}{\lim \sup } \frac{N(r, a)}{-\log (1-r)}=\tilde{\mu}_{a}
$$

By the First Fundamental Theorem of Nevanlinna theory we have $N(r, a) \leq T(r, f)+0(1)$, $(r \rightarrow 1)$, and so $0 \leq \tilde{\mu}_{a} \leq \tilde{\alpha}_{\text {. }} \tilde{\mu}_{\infty}$ is defined similarly.

THEOREM 4.4. Let $f$ be a meromorphic function in $D$ for which $1<\tilde{\alpha}<+\infty$ with $\tilde{\alpha}$ defined by (4.1). Then for every $a$ in $u\{\infty\}$ with at most $q-1$ exceptions, where $q \geq 3$,

$$
\tilde{\mu}_{a} \geq(1 / q)((q-2) \tilde{\alpha}-1) \text {. }
$$

In SECTION TWO we introduced the Nevanlinna deficiency and the index of multiplicity. Using the notation established there, we have the following theorem related to THEOREM 2.10.

THEOREM 4.5. Let $f$ be a meromorphic function in $D$ such that $0<\tilde{l}<+\infty$ where $\tilde{l}$ is defined by (4.2). Then the set of values a in $\mathcal{C} U\{\infty\}$ for which $\Theta(a)>0$ is countable,

$$
\sum_{a}\{\delta(a)+\theta(a)\} \leq \sum \Theta(a) \leq 2+\frac{1}{\tilde{l}} . \quad \text { (Sons [4]). }
$$

An immediate corollary to THEOREM 4.5 presents a limitation on the number of values such a function can take only a finite number of times.

THEOREM 4.6. Let $f$ be a meromorphic function in $D$ such that $0<\tilde{\ell}<+\infty$ where $\tilde{\ell}$ is defined by $(4,2)$. If

$$
\tilde{\ell}>1 /(p-2) \text {, }
$$


where $p$ is an integer larger than 2, then $f$ cannot have $p$ values taken only a finite number of times.

In fact, it is true that the above theorem holds if $\tilde{\ell}$ is replaced by $\tilde{\alpha}$. Ve have

THEORE?: 4.6'. Let $f$ be a meromorphic function in $D$ such that $0<\tilde{\alpha}<+\infty$ where $\tilde{\alpha}$ is defined by $(4,1)$. If

$$
\tilde{\alpha}>1 /(p-2),
$$

where $p$ is an integer larger than 2, then $f$ cannot have $p$ values taken only a finite number of times (Tsuji [3, p.215]).

THEOREMS 4.6 and $4.6^{\prime}$ rely on the fact that when such $f$ take on a value a only a finite number of times, then $\delta(a)=1$. These theorems are best possible. For each integer $p$ with $p \geq 3$, we consider an automorphic function $g$ which maps the unit disc onto the universal convering surface of the plane punctured at the $p$ points $a_{1}, a_{2}, a_{3}, \ldots, a_{p}$. For $g$ we have $\tilde{l}=\tilde{\alpha}=1 /(p-2)$, (Nevanlinna $[6, p, 272]$ and $[71, \mathrm{p} .153])$.

An immediate corollary to THEOREM $4.6^{\prime}$ is the following.

THEOREM 4.7. Let $f$ be a meromorphic function in $D$ such that $0<\tilde{\alpha}<+\infty$ where $\tilde{\alpha}$ is defined by (4.1). Then $f$ cannot omit an infinite number of values.

The techniques used in proving THEOREMS 2.17 and 2.18 make it possible to establish some theorems concerning the value distribution of derivatives of functions in our class.

THEOREM 4.8. Let $f$ be a meromorphic function in $D$ such that $3<\tilde{\alpha}<+\infty$ where $\tilde{\alpha}$ is defined by $(4.1)$. If

$$
N(r, f)=o(T(r, f)), \quad(r \rightarrow 1),
$$

and

$$
N(r, 1 / f)=0(T(r, f)), \quad(r \rightarrow 1),
$$

then $f^{\prime}$ assumes every finite complex value except possibly zero infinitely of ten (Sons [4]).

If one is willing to take $\tilde{\alpha}$ larger, similar conclusions can be made for higher order derivatives. 
An analogue of THEOREM 2.18 is obtained by consideration of the lower growth of the derivatives.

TEEOREM 4.9. Let $f$ be a meromorphic function in $D$ such that $0<\tilde{\alpha}<+\infty$ where $\tilde{\alpha}$ is defined by (4.1). Let $v$ be a positive integer, and let

$$
\begin{aligned}
& \ell_{v}=\underset{r \rightarrow 1}{\lim \inf } \frac{T\left(r, f^{(v)}\right)}{-\log (1-r)} . \\
& \sum_{a \neq \infty}^{\Theta\left(a, f^{(v)}\right) \leq 1+\frac{1}{v+1}+\frac{1}{l_{v}}}
\end{aligned}
$$

Then

In particular, $f^{(\nu)}$ assumes every finite value with at most one exception infinitely of ten provided $\ell_{v}>(v+1) / v$ (Sons [4]).

Turning to consideration of asymptotic values, we observe that THEOREMS 2.24 and 2.26 have the following as a corollary.

THEOREM 4.10. Let $f$ be a non-constant meromorphic function in $D$, with $\tilde{\alpha}<+\infty$ where $\tilde{\alpha}$ is defined by (4.1). Suppose there exists an a in $\mathbb{C} \cup\{\infty\}$ such that

$$
N(r, a)=0(1), \quad(r \rightarrow 1)
$$

Then

(i) $\quad$ f $\in A_{m}$, (Barth [31]);

(ii) for $n>0$, either $f^{(n)}$ is in $A_{m}$ or $f^{(n)}$ is a constant function (Barth and Schneider [32]).

If one considers the class $F$ of all meromorphic functions $f$ in $D$ such that $\tilde{\alpha}<+\infty$ where $\tilde{\alpha}$ is defined by $(4.1)$, an important subclass is those functions which are normal in $D$.

0. Lehto and K. Virtanen [27] have shorn that if $f$ is a normal, meromorphic function in $D$, then

$$
T(r, f) \leq K \log (1 /(1-r))+\eta(1), \quad(r \rightarrow 1)
$$

where $\mathrm{K}$ is a positive constant, Further, it 1s known (c.f. Hayman and Storvick [72]) that the derivative of a normal function need not be a normal function, but 1 t cannot grow too rapidly either according to THEOREM 4.1 . 
One interesting property of normal functions is how asymptotic values at points of the unit circle are related to angular limits at such points.

THEOREM 4.11. If $f$ is a normal meromorphic function in $D$ and has the asymptotic value a along an arc in $D$ ending at $\zeta,|\zeta|=1$, then $f$ has the angular limit a at $\zeta$ (Lehto and Virtanen [27]).

For normal analytic functions another value distribution result can be stated.

THEOREM 4.12. Let $f$ be a normal analytic function in $D$, Then either

(i) $f$ has finite radial limits on a dense subset of $\{z|| z \mid=1\}$;

or

(ii) $f$ assumes every complex number infinitely often (Sons [73]).

Other interesting examples of functions in $F$ can be found in [42] and [59]. M. Tsuji [74] has shown that certain automorphic functions are in $F$. Also the derivatives of some functions of bounded Nevanlinna characteristic are not of bounded characteristic but are in $F$ (with $\tilde{\alpha} \leq 1$ ) (c.f. Hayman [75]).

5. SECTION FIVE.

We consider theorems concerning functions analytic in $D$ with growth more restrictive than that in SECTION THREE. For an analytic function $f$ in $D$ define $\tilde{\rho}$ and $\tilde{\lambda}$ by

$$
\lim _{r \rightarrow 1} \frac{\log ^{+} M(r, f)}{-\log (1-r)}=\tilde{\rho}
$$

and

$$
\lim \inf \frac{\log ^{+} M(r, f)}{-\log (1-r)}=\tilde{\lambda} \text {, }
$$

where $M(r, f)$ is the maximum modulus of $f$ on $\{z|| z \mid=r\}$. We have

$$
0 \leq \tilde{\lambda} \leq \tilde{\rho} \leq+\infty
$$

Subsets of the class here include univalent functions (the classical Koebe function has $\tilde{\rho}=2$ ), $\mathrm{p}$-valent functions of various types, and functions in ${ }_{\mathrm{H}^{\mathrm{p}}}$ classes $(0<p<\infty)(c, f$. Duren [76], Hayman [77], and Pommerenke [78]).

Some restriction is obtained on the growth of the derivative of the function in terms of the growth of the function.

THEOREM 5.1. Let $f$ be an analytic function in $D$ for which $\tilde{\rho}$ is defined by 
(5.1) and $\tilde{\rho}>0$, Then

$$
\tilde{\rho} \leq \tilde{\rho}^{\prime}=1 \lim _{r \rightarrow 1} \sup \frac{\log ^{+} M\left(x, f^{\prime}\right)}{-\log (1-r)} \leq \tilde{\rho}+1
$$

The funtion $f$ defined in $D$ by $f(z)=(1-z)^{-1}$ has $\tilde{\rho}=1$ and $\tilde{\rho}^{\prime}=2$.

The growth of functions in this class is reflected by the power series structure of the functions as follows:

THEOREI 5.2. Let $f$ be an analytic function in $D$ for which $\tilde{\rho}$ is defined by (5.1) and $\tilde{\rho}>0$. If

$$
f(z)=\sum_{n=0}^{\infty} a_{n} z^{n}, \quad(z \in D)
$$

then $\beta_{0}=\beta \leq \tilde{\rho}$, where

$$
B_{0}=\lim \sup _{n \rightarrow \infty} \frac{\log ^{+}\left|a_{n}\right|}{\log n} \text {, and } B=\lim \sup _{r \rightarrow 1} \frac{\log ^{+} \mu(r)}{-\log (1-r)} \text {. }
$$

(c.f. Sons [73]).

The function $f$ defined in $D$ by $f(z)=(1-z)^{-1}$ has $\beta_{0}=0$ and $\tilde{\rho}=1$, so the inequality may occur.

Inequality (3.4) gives (the easy) THEOREM 5.3.

THEOREM 5.3. Let $f$ be an analytic function in $D$ for which $\tilde{\rho}$ is defined by (5.1). Then

$$
\tilde{\alpha} \leq \tilde{\rho}
$$

where $\tilde{\alpha}$ is defined by $(4.1)$.

An adaptation of a construction of $J$. Clunie [79] enables one to obtain many functions in the class.

THEOREM 5.4. Let $\phi$ be an increasing, convex, continuous function defined on the interval $[0,1)$ for which

$$
\lim _{r \rightarrow 1} \phi(r)=+\infty .
$$

Then there exists an analytic function $f$ in $D$ with

(i) $\log M(r, f) \sim \phi(r), \quad(r+1)$;

(ii) $T(r, f) \sim \phi(r), \quad(r \rightarrow 1)$. 
Using Jensen's formula, one can get the following bound on zeros for a function satisfying $(5,1)$ with $\tilde{\rho}<+\infty$,

THEOREM 5.5, Let $f$ be a function which is analytic in $D$. Assume $\tilde{\rho}$ is defined by $(5,1)$ and $\tilde{\rho}<+\infty$. Then

$$
\underset{r \rightarrow 1}{\lim \sup } \frac{(1-r) n(r, 1 / f)}{-\log (1-r)} \leq \tilde{\rho} .
$$

An example in [59] shows that the left-hand side of (5.3) may be positive. THEOREMS 3.15 and 3.15 show the zeros of the example must be spread around the disc.

If $f$ grows in a regular enough way, some additional results can be obtained.

If $f$ is an analytic function in $D, f$ is said to be in $A^{-n}$ if and only if

$$
|f(z)| \leq C(1-|z|)^{-n},(z \in D),
$$

where $C$ is a positive constant and $0<n<\infty$. H. Shapiro and A. Shields [59] note that these are precisely the functions for which their Taylor series representation

$$
\sum_{n=0}^{\infty} a_{n} z^{n}
$$

have

$$
\sum_{n=1}^{\infty}\left(\left|a_{n}\right|^{2}\right) / n^{k}<\infty
$$

for some $k$.

If $f$ is an analytic function in $D$, then $f$ is said to be in $A^{p}(0<p<\infty)$ if and only if

$$
\iint_{|z|<1}|f(z)|^{p} d x d y<\infty .
$$

(For $\mathrm{p}=2$, one gets the "Bergman space").

E. Beller [56]points out that for all positive $p$ and $n$, the following inclusions hold:

$$
A^{P} \subset A^{-2 / P}
$$

and for all $\varepsilon>0$,

$$
A^{-n} \subset A^{(1 / n)+\varepsilon} .
$$


For functions in $A^{P}$ the following can be proved,

THEOREM 5.6, If $f$ is an analytic function in $D$ which is also in $A^{P}(0<p<\infty)$ and if $\left\{a_{k}\right\}$ are the zeros of $f$ arranged so that

$$
\left|a_{1}\right| \leq\left|a_{2}\right| \leq\left|a_{3}\right| \leq \ldots
$$

then

$$
\lim \sup _{r \rightarrow 1} \frac{(1-r) n(r, 1 / f)}{-\log (1-r)} \leq \frac{1}{p}
$$

and

$$
\lim _{n \rightarrow \infty} \frac{1-\left|a_{n}\right|}{n^{-1} \log n} \leq \frac{1}{p} .
$$

Further, those results are sharp (Beller [57] and Horwitz [80]).

In [81] B. Korenblum has given a necessary condition for a subset of $D$ to be a zero set of $A^{-n}$; he has also given a sufficient condition for a subset of $D$ to be a zero set of $A^{-n}$ and a necessary and sufficient condition for a subset of $D$ to be a zero set of

$$
A^{-\infty}=u_{n>0} A^{-n} .
$$

(Every $f$ in $A^{-\infty}$ has the form

$$
f(z)=\sum_{k=0}^{\infty} c_{k} z^{k}
$$

with

$$
c_{k}=0\left(k^{n}\right), \quad(k \rightarrow \infty)
$$

for some $n$ ).

There are functions defined in $D$ by gap power series with gaps smaller than (3.11) and growth not as rapid as that implied by (3.13) for which conclusions of the nature of THEOREY 3.36 can be made. For example,

THEOREM 5.7. Let $f$ be an analytic function in $D$ defined by the power series representation (3.10) where the sequence $\left\{n_{k}\right\}$ satisfies 


$$
\sum_{\substack{k=0 \\ k \neq v}}^{\infty} \frac{n_{v}^{2}}{\left(n_{k}-n_{v}\right)^{2}}=0\left\{\left(\log n_{v}\right)^{2}\right\}, \quad(v+\infty)
$$

If $\tilde{\rho}>0$ where $\tilde{\rho}$ is defined by $(5,1)$, then,

(i) for each complex number $a, \delta(a, f)=0$; in fact

$$
\underset{r \rightarrow 1}{\lim \sup }(N(r, 1 /(f-a)) /(\log M(r, f))=1 ;
$$

(Nicholls and Sons [68]).

and

(ii) $f$ has no finite asymptotic values (Sons [82]).

6. SECTION SIX.

In the earlier sections we have considered functions $f$ analytic or meromorphic in $D$ for which either $T(r, f)$ or $M(r, f)$ is unbounded as $r$ approaches one. Some of the results stated in those sections apply to functions $f$ which grow less rapidly than having $\alpha$ or $\rho$ or $\tilde{\alpha}$ or $\tilde{\rho}$ positive, where these are defined by $(2.1),(3,1)$, (4.1), and (5.1) respectively. There are some other results known for functions of slow growth.

In [71] R. Nevanlinna indicates that if a function $f$ omits an infinite number of values subject to certain special conditions, then

$$
T(r, f)=0\left(\log \log \frac{1}{1-r}\right), \quad(r \rightarrow 1) .
$$

The set of Bloch functions, a subset of the set of normal functions introduced in SECTION TWO, has slow growth. An analytic function $f$ in $D$ is called Bloch if

$$
\sup _{z \in D}\left(1-|z|^{2}\right)\left|f^{\prime}(z)\right|<\infty \text {. }
$$

We have

THEOREM 6.1. Let $\mathrm{f}$ be an analytic function in $\mathrm{D}$ which is a Bloch function. Then

$$
\underset{r \rightarrow 1}{\lim \sup } \frac{N(r, 1 / f)}{\log \log (1 /(1-r))} \leq \underset{r \rightarrow 1}{\lim \sup } \frac{T(r, f)}{\log \log (1 /(1-r))} \leq \frac{1}{2} .
$$

Conversely, there exists a Bloch function such that equality holds in (6.1) 
(Anderson, Clunie, and Pommerenke [83] and offord [84]),

In recent years annular functions have been studied concerning their value distribution (c.f. Bagemihl and Erdōs [85], Bonar [86], and Bonar and Carrol1 [87]). An analytic function $f$ in $D$ is said to be anmular if there exists a sequence $\left\{J_{n}\right\}$ of Jordan curves in $D$ such that

(i) $\mathrm{J}_{\mathrm{n}}$ lies in the interior of $\mathrm{J}_{\mathrm{n}+1}$.

(ii) for each $\varepsilon>0$ there exists a number $n_{0}$ such that, for $\mathrm{n}>\mathrm{n}_{0}, \mathrm{~J}_{\mathrm{n}}$ lies in the region $\{\mathrm{z}|1-\varepsilon<| \mathrm{z} \mid<1\}$, and (iii) $\phi\left(J_{n}\right)=\min \left\{\mid f(z) \| z \in J_{n}\right\} \rightarrow \infty$ as $n \rightarrow \infty$.

THEOREM 6,2, Let $f$ be an annular function in $D$ with defining curves $\left\{J_{n}\right\}$ as above. Let $\left\{r_{n}\right\}$ be a sequence of numbers increasing to one such that $J_{n}$ is contained in $\left\{z|| z \mid \leq r_{n}\right\}$, Then

$$
\underset{n \rightarrow \infty}{\lim \inf } \frac{N\left(r_{n}, 1 / f\right)}{\log \phi\left(J_{n}\right)} \geq 1
$$

(Nicholls and Sons [68]).

Another growth indicator for functions $f$ analytic or meromorphic in $D$ is $m_{2}(r, f)$ defined by

$$
m_{2}(r, f)=\left\{-\frac{1}{2 \pi} \int_{0}^{2 \pi}\left(\log \left|f\left(r e^{i \theta}\right)\right|\right)^{2} d \theta\right\}^{1 / 2},(0<r<1) .
$$

A number of properties of $m_{2}(r, f)$ and some value distribution theorems involving $m_{2}(r, f)$ appear in [88]. In [89] some additional results are obtained with special consideration given to the case where $m_{2}(r, f)$ approaches infinity as $r$ approaches one, but $T(r, f)$ is bounded. 


\section{REFERENCES}

1. HAYMAN, W.K, Meromorphic Functions, Oxford, London, 1964.

2. TSUJI, M. Canonical product for a meromorphic function in the unit circle, J. Math, Soc. Japan 8 (1956), 7-21.

3. TSUJI, M. Potential Theory in Modern Function Theory, Maruzen, Tokyo, 1959.

4. SONS, L.R. Value distribution for the derivatives of functions with slow growth in the disc, preprint.

5. TSUJI, M. Borel's direction of a meromorphic function in the unit circle, J. Math. Soc. Japan 7 (1955), 290-311.

6. NEVANLINNA, R. Eindeutige analytische Funktionen, Second Edition, Springer-Verlag, Berlin, 1953,

7. DZHRBASHYAN, M.M. The theory of factorization and boundary properties of functions meromorphic in a disc, Russian Math. Surveys 28 (1973), 1-12.

8. GIRNYK, M.A. Ein Analogon eines Satzes von Lindelöf über den Typ kanonischer Produkte, Teor. Funkts. Funkts. Anal. Prilozh. 29 (1978), 16-24.

9. GIRNYK, M.A. Asymptotic properties of some canonical products II, Siberian Math. J. 17 (1976), 718-731. Correction Siberian Math. J. 18 (1977), 510.

10. SONS, L.R. Borel exceptional values in the unit disc, Rocky Mountain J. Math. 12 (1982), 197-204.

11. SONS, L.R. Value distribution of canonical products in the unit disc, Math. Japonica 22 (1977), 27-38.

12. LITTLEWOOD, J.E. Mathematical Notes (II): On exceptional values of power series, J. London Math. Soc. 5 (1930), 82-87

13. AHLFORS, L. Ein Satz von Henri Cartan und seine Anvendung auf die Theorie der meromorphen Funktionen, Soc. Sci. Fenn. Comm. Phys. Math. 16 (1931), 1-19.

14. HAYMAN, W.K. The proximity function in Nevanlinna theory, J. London Math. Soc. (2), 25 (1982), 473-482.

15. KRUTIN, V.I. The size of Nevanlinna deficiencies of functions meromorphic in $|z|<1$, Izv. Akad. Nauk Armjan. SSSR Ser. Mat. $\underline{8}$ (1973), 347-358.

16. GIRNYK, M.A, On the inverse problem of the theory of the distribution of values for functions that are analytic in the unit disc, Ukrainian Math. J. 29 (1977), 24-30.

17. HAYMAN, W.K. On Nevanlinna's second theorem and extensions, Rend. Circ. Mat. Palermo (2) $\underline{2}$ (1953), 1-47.

18. WITTICH, H. Neuere Untersuchungen uber eindeutige analytische Funktionen, Zneite korrigierte Auflage, Springer-Verlag, Berlin, 1968.

19. HAYMAN, W.K. Picard values of meromorphic functions and their derivatives, Annals of Math. (2) 70 (1959), 9-42.

20. VALIRON, G, Fonctions analytiques et equations differentielles, J. Math pures et app1. 31 (1952), 292-303. 
21. BANK, S, A general theorem concerning the growth of solutions of first-order algebraic differential equations, Compositio Math. 25 (1972), 61-70.

22. BANK, S. On solutions of algebraic differential equations whose coefficients are analytic functions in the unit disc, Ann. Mat. Pura. Appl. 92 (1972), 323-335.

23. BANK, S. On the existence of meromorphic solutions of differential equations having arbitrarily rapid growth, J. Reine Angew. Math. 288 (1976), 176-182.

24. GOL'DBERG, A.A. Growth of functions meromorphic in a disc with restrictions on the logarithmic derivative, Ukrainian Math. J. 32 (1980), 311-316.

25. GOL'DBERG A.A. On single-valued solutions of first order differential equations, Ukrain. Mat. Zh. 8 (1956), 254-261.

26. MACLANE, G. R. Meromorphic functions with small characteristic and no asymptotic values, Michigan Math. J. 8 (1961), 177-185.

27. LEHTO, 0. and VIRTANEN, K.I. Boundary behavior and normal meromorphic functions, Acta Math. 97 (1957), 47-65.

28. CARTWRIGHT, M.L. and COLLINGWOOD, E.F. Boundary theorem for a function meromorphic in the unit circle, Acta Math. 87 (1952), 83-146.

29. CARTWRIGHT, M.L. and COLLINGWOOD, E.F. The radial limits of functions meromorphic in a circular disc, Math. Z. 76 (1961), 404-410.

30. BARTH, K.F. and SCHNEIDER, H.J. On a problem of Collingwood concerning meromorphic functions with no asymptotic values, J. London Math. Soc. (2) 1 (1969), 553-560.

31. BARTH, K.F. Asymptotic values of meromorphic functions, Mich. Math. J. 13 (1966), 321-340.

32. BARTH, K.F. and SCHNEIDER, W.J. Integrals and derivatives of functions in MacLane's class A and of normal functions, Proc. Camb. Phil. Soc. 71 (1971), 111-121.

33. BOAS, R. Entire Functions, Academic Press, New York, 1954.

34. MACLANE, G.R. Asymptotic values of holomorphic functions, Rice Univ. Studies $49(1963), 1-83$.

35. KAPOOR, G.P. On the lower order of functions analytic in the unit disc, Math. Japon. 17 (1972), 49-54.

36. KAPOOR, G.P. and JUNEJA, O.P. On the lower order of functions analytic in the unit disc II, Indian J. Pure App1. Math. 7 (1976), 241-248.

37. SONS, L.R. Regularity of growth and gaps, J. Math. Anal. Appl. 24 (1968), 296-306. Corrigendum, J. Math. Ana1. App1. 58 (1977), 232 .

38. SHEA, D,F. Functions analytic in a finite disc and having asymptotically prescribed characteristic, Pacific J. Math. 17 (1966), 549-560.

39. LINDEN, C.N. Functions analytic in a disc having prescribed asymptotic growth properties, J. London Math. Soc, (2) 2 (1970), 267-272.

40, LINDEN, C.N. The representation of regular functions, J. London Math. Soc. 39 (1964), 19-30. 
41. LINDEN, C.N. The distribution of the zeros of regular functions, Proc. London Math. Soc. (3) 15 (1965) 301-322.

42. BAGEMIHL. F.; ERDÖS, P. and SEIDEL, W. Sur quelques propriétés frontiefes des fonctions holomorphes defines par certains prodults dans le cercleunité, Ann. Sct. Ecole Norm. Sup. (3) 70 (1953), 135-147.

43. MACLANE, G.R. holomorphic functions, of arbitrarily slow growth, without radial limits, Mich. Math. J. 9 (1962), 21-24.

44. BAGEMIHL, F. Curvilinear cluster sets of arbitrary functions, Proc. Nat. Acad. Sci. U.S.A. 41 (1955) 379-382.

45. HEINS, M. A property of the asymptotic spots of a meromorphic function or an interior transformation whose domain is the open unit disk, J. Indian Math. Soc. 24 (1960), 265-268.

46. HORNBLOWER, R. A growth condition for the MacLane class A, Proc. London Math. Soc. (3) 23 (1971), 371-384.

47. MACLANE, G.R. A growth condition for class A, Mich. Math. J. 25 (1978), 263-287.

48. RIPPON, P.J. On a growth condition related to the Maclane class, J. London Math. Soc. (2) $18(1978), 94-100$.

49. GROSZ, W. Eine ganze Funktion, fur die jede komplexe Zah1 Konvergenzwert ist, Math. Ann. 79 (1918), 201-208.

50. DRASIN, D. Some problems related to MacLane's class, J. London Math. Soc. (2) $23(1981), 280-286$.

51. MCMILLAN, J.E. Asymptotic values of functions holomorphic in the unit disc, Mich. Math. J. 12 (1965), 141-154.

52. LINDEN, C.N. The minimum modulus of functions regular and of finite order in the unit circle, Cuart. J. Math. Oxford (2) 7 (1956), 196-216.

53. LINDEN, C.N. Minimum modulus theorems for functions regular in the unit circle, Quart. J. Math. Oxford (2) 12 (1961), 1-16.

54. LINDEN, C.N. "The minimum modulus of functions of slow growth in the unit disk," Mathematical Essays Dedicated to A.J. MacIntyre, Ohio University Press, Athens (Ohio), 1970.

55. ARIMA, K. On maximum modulus of integral functions, J. Math. Soc. Japan 4 (1952), 62-66.

56. BELLER, E. Factorization for non-Nevanlinna classes of analytic functions, Israel J. Math. 27 (1977), 320-330.

57. BELLER, E. Zeros of $A^{p}$ functions and related classes of analytic functions, Israel J. Math. 22 (1975), 68-80.

58. HEILPER, A. The zeros of functions in Nevanlinna's area class, Israel J. Math. $34(1980), 1-11$.

59. SHAPIRO, H.S. and SHIELDS, A,L. On the zeros of functions with finite Dirichlet integral and some related function spaces, Math. Z. 80 (1962), 217-229.

60. HAYMAN, W.K. and KORENBLUM, B. A critical growth rate for functions regular in a disk, Mich. Math. J. 27 (1980), 21-30. 
61. LINDEN, C,N, Regular functions of restricted growth and their zeros in tangential regions, preprint.

62. HANSON, B. $\mathrm{Ph}, \mathrm{D}$. Dissertation, University of Wisconsin, preprint.

63. MURAI, T. The value distribution of lacunary series and a confecture of Paley, Ann. Inst. Fourier (Grenoble) 31 (1981), 136-156.

64. MURAI, T, Sur la distribution des valeurs des sêries lacunaires, J. London Math. Soc. (2) $21(1980), 93-110$.

65. MURAI, T. The boundary behavior of Hadamard lacunary series, Nagoya Math. J. 89 (1983), to appear.

66. WIMAN, A. Über den Zusammenhang zwischen dem Maximalbetrage einer analytischen Funktion und dem grössten Betrage bei gegebenen Argumente der Funktion, Acta. Math. 41 (1916), 1-23.

67. SONS, L.R. Gap series with rapid growth in the unit disc, Math. Z. 131 (1973), 25-48.

68. NICHOLLS, P.J. and SONS, L.R. Minimum modulus and zeros of functions in the unit disc, Proc. London Math. Soc. (3) 31 (1975), 99-113.

69. SONS, L.R. Value distribution and power series with moderate gaps, Mich. Math. J. $13(1966), 425-433$.

70. SONS, L.R. Asymptotic paths for power series with exponents of the form $\mathrm{n}^{\mathrm{P}}$, Mich. Math. J. 19 (1972), 383-384.

71. NEVANLINNA, R. Le Theoreme de Picard-Borel et la theorie des fonctions meromorphes, Gauthier-Villars, Paris, 1929.

72. HAYMAN, W.K. and STORVICK, D.A. On normal functions, Bull. London Math. Soc. 3. $(1971), 193-194$.

73. SONS, L.R. Gap series - growth, normality, and values assumed, Houston J. Math. I (1981), 431-440.

74. TSUJI, M. Theory of Fuchsian groups, Jap. J. Math. 21 (1951), 1-27.

75. HAYMAI, U.K. On the characteristic of functions meromorphic in the unit disk and of their integrals, Acta Math. 112 (1964), 181-214.

76. DUREN, P. Theory of $\mathrm{H}^{\mathrm{p}}$ Spaces, Academic Press, New York and London, 1970.

77. HAYMAN, W.K. Multivalent Functions, Cambridge Univ. Press, London, 1967.

78. POMMERENK, C. Univalent Functions, Vandenhoeck and Ruprecht, Göttingen, 1975.

79. CLUNIE, J. On integral functions having prescribed asymptotic growth, Canadian J. Math. 17 (1965), 396-404.

80. HOROWITZ, C. Zeros of functions in the Bergman spaces, Duke Math. J, 41 (1974), 693-710.

81. KORENBLUM, B, An extension of the Nevanlinna theory, Acta Math. 135 (1975), 187-219.

82. SONS, L.R. Zeros of power series with large gaps, Indiana Univ. Math. J. 22 (1972), 199-205. 
83. ANDERSON, J,M.; CLUNIE, J, and POMMERENKE, C. On Bloch functions and normal functions, J, reine angewand te Math. 270 (1974), 12-37.

84. OFFORD, A.C. The distribution of the values of a random function in the unit disk, Studia Math, 41 (1972), 71-106.

85. BAGEMIHL, F, and ERDÖS, P. A problem concerning the zeros of a certain kind of holomorphic function in the unit circle, $J$, reine angewandte Math. 214/215 (1965), 340-344.

86. BONAR, D.D. On Annular Functions, VEB Deutscher Verlag der Wissenschaften, Berlin, $197 \overline{1 .}$

87. BONAR, D, and CARROLL, F. Fatou points for annular functions, J. reine angewandte Math. 283/284 (1976), 222-226.

88. SONS, L,R. Zeros of functions with slow growth in the unit disc, Math. Japonica $\underline{24}$ (1979), 271-282.

89. SONS, L.R. Zero distribution of functions with slow on moderate growth in the unit disc, Pacific J. Math. 99 (1982), 473-481. 


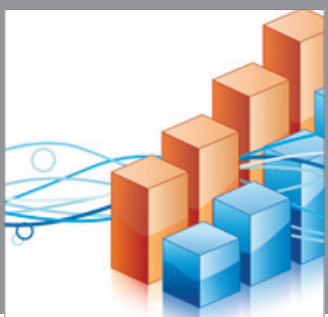

Advances in

Operations Research

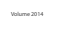

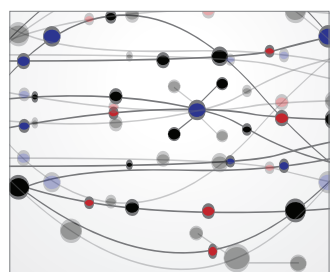

\section{The Scientific} World Journal
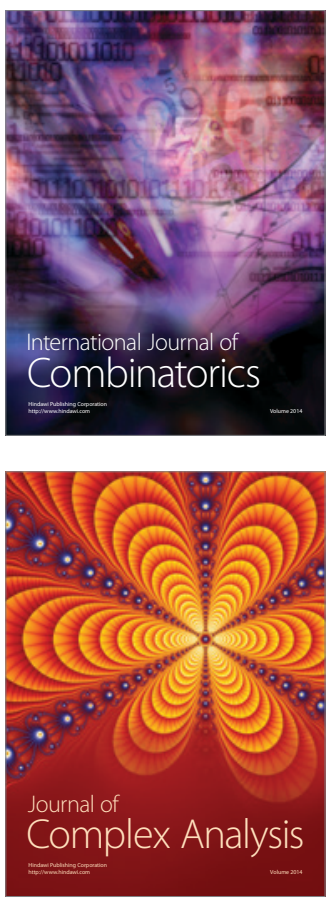

International Journal of

Mathematics and

Mathematical

Sciences
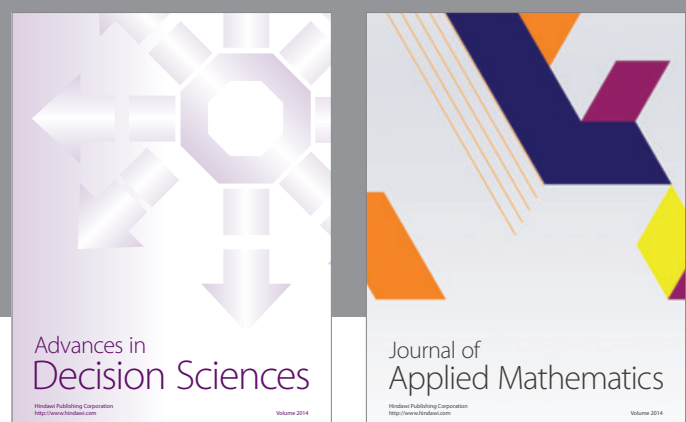

Journal of

Applied Mathematics
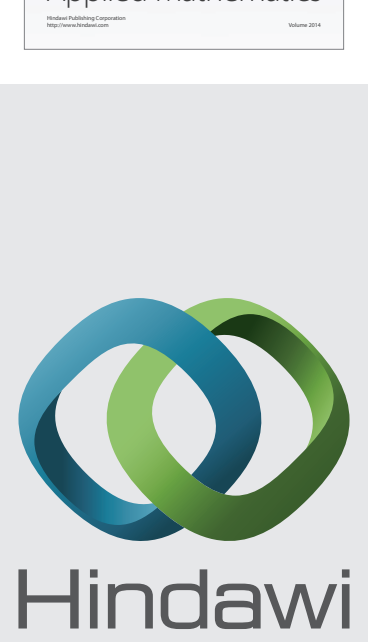

Submit your manuscripts at http://www.hindawi.com
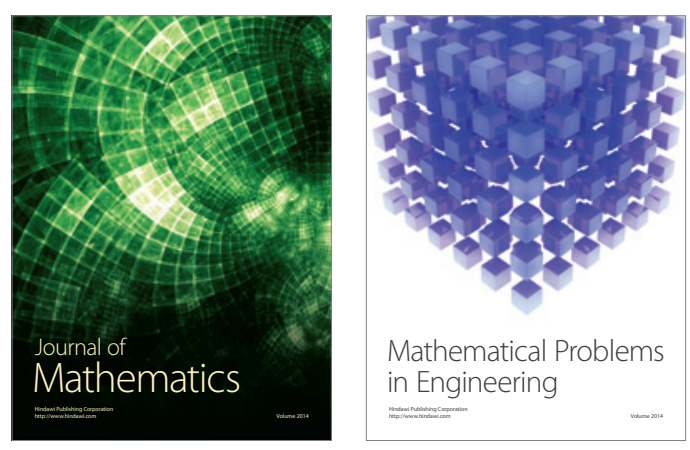

Mathematical Problems in Engineering
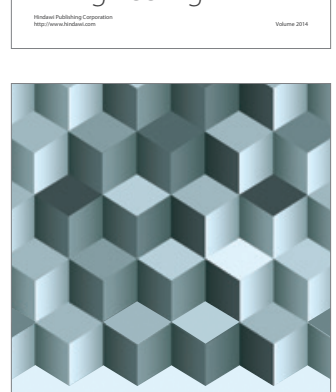

Journal of

Function Spaces
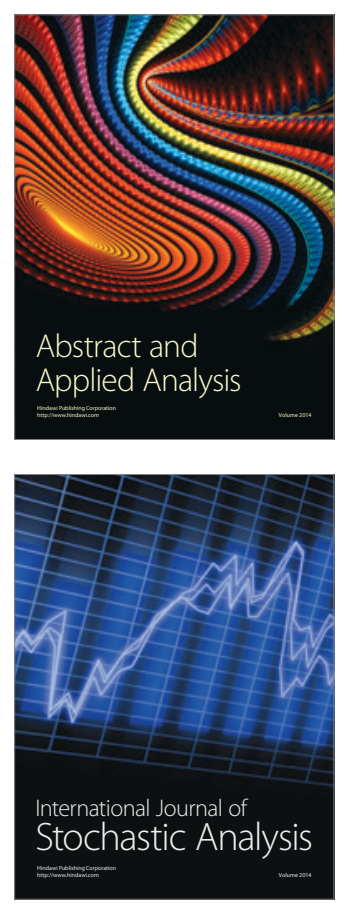

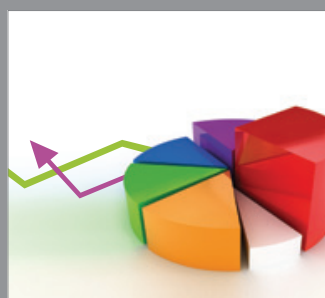

ournal of

Probability and Statistics

Promensencen
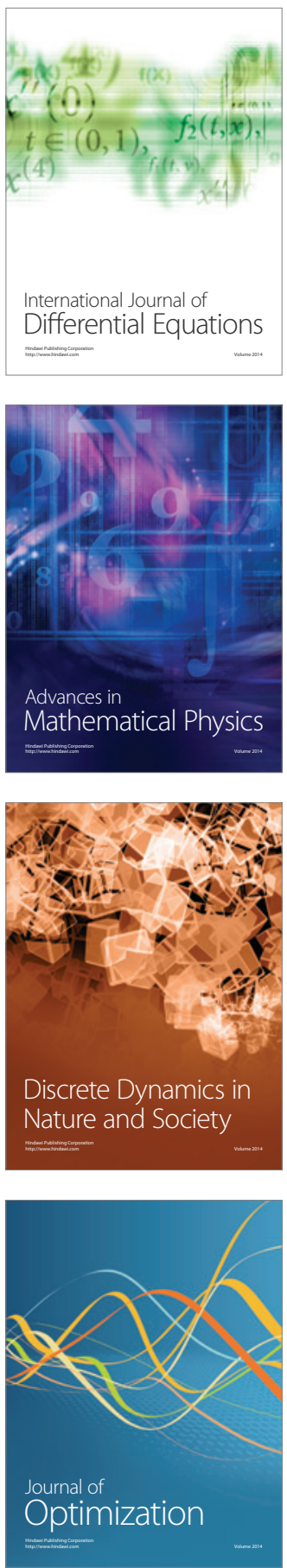